\title{
Dynamic Earthquake Ruptures in the Presence of Lithostatic Normal Stresses: Implications for Friction Models and Heat Production
}

\author{
by Brad T. Aagaard, Thomas H. Heaton, and John F. Hall
}

\begin{abstract}
We simulate dynamic ruptures on a strike-slip fault in homogeneous and layered half-spaces and on a thrust fault in a layered half-space. With traditional friction models, sliding friction exceeds $50 \%$ of the fault normal compressive stress, and unless the pore pressures approach the lithostatic stress, the rupture characteristics depend strongly on the depth, and sliding generates large amounts of heat. Under application of reasonable stress distributions with depth, variation of the effective coefficient of friction with the square root of the shear modulus and the inverse of the depth creates distributions of stress drop and fracture energy that produce realistic rupture behavior. This ad hoc friction model results in (1) low-sliding friction at all depths and (2) fracture energy that is relatively independent of depth. Additionally, friction models with rate-weakening behavior (which form pulselike ruptures) appear to generate heterogeneity in the distributions of final slip and shear stress more effectively than those without such behavior (which form cracklike ruptures). For surface rupture on a thrust fault, the simple slip-weakening friction model, which lacks rate-weakening behavior, accentuates the dynamic interactions between the seismic waves and the rupture and leads to excessively large ground motions on the hanging wall. Waveforms below the center of the fault (which are associated with waves radiated to teleseismic distances) indicate that source inversions of thrust events may slightly underestimate the slip at shallow depths.
\end{abstract}

\section{Introduction}

A better understanding of the rupture process provides an important avenue for improving the models of nearsource ground motions and gaining insight into the physics of earthquakes. Including the rupture dynamics in simulations of earthquakes generally involves modeling the frictional sliding on the fault surface, with two distinct efforts having emerged in recent years. Those researchers who model the evolution of stress on the fault almost exclusively use state- and rate-dependent friction models. Review articles by Marone (1998) and Scholz (1998) summarize the development of the friction models and some of the features of their behavior. These models are based on laboratory experiments of sliding at slip rates between $10^{-7}$ and $1 \mathrm{~mm} /$ sec and can be derived from analytical models of creep behavior (Persson, 1997). Consequently, researchers apply these models to studies of the nucleation of earthquakes and creep behavior on faults (e.g., Stuart and Mavko, 1979; Rice and Ben-Zion, 1996; Tullis, 1996). As the coefficient of friction in these state- and rate-dependent friction models is typically around 0.6 , their application to dynamic ruptures predicts large temperature changes in the zone surrounding the fault, unless the dynamic compressive stresses on the fault are much less than the lithostatic pressures (Richards, 1976; Kanamori and Heaton, 2000).

The other effort focuses on modeling the rupture behavior during earthquakes. The uncertainty in the behavior of how faults rupture has led researchers to create simple, ad hoc models that produce reasonable behavior. These models generally include either slip-weakening behavior (the shear strength decreases as slip occurs) or a combination of slip weakening and rate weakening (initially, the shear strength drops with slip in response to slip weakening and then returns near its original level as the slip rate decreases). For nearly $30 \mathrm{yr}$, the slip-weakening friction model has been used to study the frictional sliding associated with earthquakes. Ida (1972) was one of the first to associate slipweakening behavior with the propagation of shear cracks. Andrews (1976a) and Burridge et al. (1979) used slipweakening friction models to study the propagation of modeII shear cracks.

At about the same time, the finite-difference and finiteelement methods were applied to the study of three-dimensional dynamic ruptures (Madariaga, 1976; Archuleta and Day, 1980; Day, 1982a). This marked a dramatic improve- 
ment in the applicability of the methods used to model dynamic ruptures because they can be used for three-dimensional simulations with heterogeneous material properties; however, the computing power at the time severely limited the size and scope of the calculations.

More recently, with the advances in computing, many more researchers have employed boundary-integral, finitedifference, or finite-element formulations to model dynamic ruptures (Mikumo, 1992; Mikumo and Miyatake, 1993; Madariaga and Cochard, 1996; Harris and Day, 1997; Olsen et al., 1997; Fukuyama and Madariaga, 1998; Oglesby et al., 1998; Harris and Day, 1999; Magistrale and Day, 1999; Nielsen and Olsen, 2000; Oglesby et al., 2000a,b). Simulations of the 1992 Landers earthquake by Olsen et al. (1997) and the 1994 Northridge earthquake by Nielsen and Olsen (2000) demonstrated the ability of the finite-difference method and slip-weakening friction models and slip- and rateweakening friction models, respectively, to produce reasonable rupture behavior. The simulations generated a confined rupture pulse consistent with the kinematic source inversions and reproduced the main long-period features of the waveforms. Using similar methods, Harris and Day (1999) and Magistrale and Day (1999) explained the propagation across step-overs between parallel strands on strike-slip and thrust faults, respectively. Additionally, Oglesby et al. studied the difference between ruptures on normal faults and thrust faults using three-dimensional (Oglesby et al., 2000a,b) finite-element simulations in a homogeneous medium.

In contrast to the simulations using state- and ratedependent friction models, only a few of the simulations performed to date with slip-weakening or slip- and rateweakening friction models assume effective normal stresses that correspond to the overburden pressure. Although Mikumo (1992) used normal stresses equal to the overburden pressure, the distributions of final slip from the dynamic ruptures exhibit a clear depth dependence, which does not match the distributions found in kinematic source models (Heaton, 1990; Somerville et al., 1999). Consequently, we examine what constraints on the friction model may be required to produce realistic ruptures when we apply reasonable normal stress distributions with depth. Using this implementation of the earthquake source and following the constraints imposed on the friction model, we determine the sensitivity of the rupture behavior and the ground motions to systematic variations of the initial shear stresses and the friction model. Additionally, we compare the ground motions from the dynamic rupture simulations with the corresponding cases of prescribed ruptures. Aagaard (1999) includes an expanded discussion of dynamic ruptures in both homogeneous and layered half-spaces.

\section{Methodology}

We use the same general solution techniques, which are described by Aagaard (1999) and Aagaard et al. (2001), to simulate the earthquakes with dynamic ruptures that we use for prescribed ruptures. Applying the finite-element method with linear tetrahedral elements to the three-dimensional dynamic elasticity equation produces the matrix differential equation,

$$
[\mathbf{M}]\{\ddot{\mathbf{u}}(t)\}+[\mathbf{C}]\{\dot{\mathbf{u}}(t)\}+[\mathbf{K}]\{\mathbf{u}(t)\}=\{\mathbf{F}(t)\},
$$

where $[\mathbf{M}],[\mathbf{C}]$, and $[\mathbf{K}]$ denote the mass, damping, and stiffness matrices, respectively, $\{\mathbf{u}(t)\}$ denotes the displacement vector at time $t$, and $\{\mathbf{F}(t)\}$ denotes the force vector at time $t$. We model the slip on the fault using sliding degrees of freedom (see Aagaard, 1999; Aagaard et al., 2001 for details) which create dislocations on the fault surface. On the fault surface we transform the usual three translational degrees of freedom on each side of the fault to six fault degrees of freedom consisting of three relative degrees of freedom (two tangential to the fault plane and one normal to the fault plane) and three average degrees of freedom (two tangential to the fault plane and one normal to the fault plane). This transformation provides explicit control of the relative motion between the two sides of the fault. In prescribed ruptures the specified slip time histories dictate the relative tangential displacements of the fault degrees of freedom, whereas in dynamic ruptures the friction model constrains the forces acting on the relative tangential fault degrees of freedom. Incorporating the fault surface into the geometry of the finite-element model allows arbitrary orientation of the fault plane.

The seismic waves generated by the rupturing fault create dynamic stresses in the surrounding volume as well as changes in the static stresses. We assume that the static stresses on the boundary of the domain remain constant during the earthquake (see the Appendix for a discussion on how this affects the energy balance). We do not need to know the initial stresses throughout the domain to model the seismic-wave propagation. However, in order to simulate the dynamic rupture of the fault, we must know the initial stresses acting on the fault surface. These stresses may be found in a number of ways, including solution of a static problem, solution of a viscoelastic problem, extrapolated from data, or assumed from intuition. Regardless of their source, we resolve the stresses into shear and normal tractions acting on the fault surface. Thus, off the fault surface we consider only the dynamic stresses and the change in the static stresses, whereas on the fault surface we also consider the initial (static) stresses.

We treat the friction on the fault as an external force and replace the force vector in the governing equation (equation 1) with the difference between the vector of tectonic forces, $\left\{\mathbf{F}_{\mathrm{t}}\right\}$, and the friction force vector, $\left\{\mathbf{F}_{\mathrm{f}}\right\}$; this yields

$[\mathbf{M}]\{\ddot{\mathbf{u}}(t)\}+[\mathbf{C}]\{\dot{\mathbf{u}}(t)\}+[\mathbf{K}]\{\mathbf{u}(t)\}=\left\{\mathbf{F}_{\mathrm{t}}(t)\right\}-\left\{\mathbf{F}_{\mathrm{f}}(D(t), \dot{D}(t))\right\}$,

where $D$ denotes the slip on the fault. As outlined previously, we only apply the tectonic forces to the fault degrees of freedom. The appearance of the difference between the tec- 
tonic force vector and the friction force vector in the equation of motion implies that we may create the same sliding behavior from an infinite combination of tectonic and friction forces by keeping the difference between them the same.

The vector $\left\{\mathbf{F}_{\mathrm{f}}\right\}$ acts on the relative tangential fault degrees of freedom, whereas the vector $\left\{\mathbf{F}_{\mathrm{t}}\right\}$ acts on both the relative normal and relative tangential fault degrees of freedom. Following the same procedure that we use for prescribed ruptures, we integrate the differential equation using the central-difference scheme. When the coefficient of friction depends on the slip rate, computing the friction at time $t$ requires knowing the slip rate at time $t$, which we do not know. To remedy this difficulty, we assume that the time step is small enough so that the slip rate does not change significantly in a single time step. Thus, we use the slip rate at time $t-\Delta t$, instead of the slip rate at time $t$, to compute the friction force at time $t$.

We must transform the tectonic tractions applied on the fault surface into forces acting on the fault degrees of freedom. At each node on the fault, we interpolate from a given distribution of initial tractions and convert the tractions to forces using the node's tributary area on the fault plane. We assume that the fault is in equilibrium and apply the forces to the relative degrees of freedom. The friction force does not require any transformation; the product of the coefficient of friction and the force associated with the relative normal degree of freedom gives the maximum magnitude of the friction force vector acting on the relative tangential degrees of freedom. The dynamic deformation in the domain may cause variations in the normal forces acting on the fault, but we do not allow normal separation of the two sides of the fault. Furthermore, except at the ground surface, the confining pressures keep the normal stresses well within the compressive regime. We compute the dynamic normal forces at the fault degrees of freedom as part of the time-stepping procedure.

\section{Initial Tractions on Fault}

We consider gravity and plate tectonics as sources of normal stresses acting on the fault surface. In a self-gravitating, spherical Earth with only radial variations in material properties, the weight of the material generates lithostatic stresses (total stress caused by gravity) with no shear stresses and equal axial stresses (Mohr's circle degenerates into a point). In addition to shear stresses, plate tectonics also creates normal stresses on the fault surface, especially in the case of inclined faults.

The presence of water in the interstices of the granular medium can generate pore pressures that decrease the effective normal stresses. If little or no water sits in the interstices, then the effect of the pore pressures is negligible, so the effective normal stresses equal the normal stresses. In a dry, homogeneous half-space, the effective normal stresses (lithostatic stresses) increase linearly with depth $(p=\rho g z)$. If water saturates the interstices, then the pore pressures equal the hydrostatic pressures, and the effective normal stresses are the difference between the normal stresses and the hydrostatic pressures. In a saturated, permeable, homogeneous half-space, the effective normal stresses again increase linearly with depth, but at a slower rate because of the presence of hydrostatic pore pressures $\left[p=\left(\rho-\rho_{w}\right) g z\right]$. Finally, if the rock is saturated but impermeable, the pore pressures can equal the lithostatic pressures, and the effective normal stresses can become very small; in this case the material essentially floats. The existence of topography and density variations implies large shear stresses ( $>10 \mathrm{MPa})$ at depths that require large normal stresses to prevent failure. Consequently, except in localized areas, we expect the effective normal stresses to be similarly large. Researchers often use effective normal stresses that are independent of depth for simplicity (Olsen et al., 1997; Ben-Zion and Andrews, 1998; Madariaga et al., 1998), without acknowledging that assuming uniform effective normal stresses with depth implies that the pore pressures increase more rapidly than the hydrostatic pressures such that the differences between the lithostatic pressures and the pore pressures are uniform with depth.

Shear tractions on the fault generate the forces that cause slip on the fault surface. We apply the shear tractions to the relative tangential degrees of freedom in the direction of the desired slip and use an asperity (usually circular in shape) with a shear stress greater than the failure stress to start the rupture. Many factors, such as the discretization size, the failure stress, and the dynamic stress drop, influence the size of the asperity necessary to initiate a propagating rupture (Andrews, 1976b; Day, 1982b; Madariaga et al., 1998).

\section{Overview of Rupture Dynamics}

We examine the anatomy of the shear stress on the fault near the rupture front, as shown in Figure 1, to find the relationship between its features and the dynamics of the rupture. From an elasticity theory treatment of dynamic fracture mechanics (see Freund, 1990), the shear stresses increase and form a singularity just ahead of the leading edge of the rupture. After slip begins, the shear stresses decrease, and then, depending on the friction model, may or may not recover as the slip rate decreases. However, in our finiteelement models (as in all discrete models) the shear stresses remain finite with a stress concentration at the rupture front rather than a singularity.

The friction model controls the decrease in friction stress as slip progresses, and therefore the dynamic stress drop (the difference between the initial shear stress and the shear stress during sliding) as well. The rate at which the dynamic stress drop increases behind the rupture front governs the slip rate, with faster decreases in shear stress leading to greater slip rates. Additionally, a larger dynamic stress drop results in a larger stress concentration at the leading edge of the rupture. The increase in shear stress associated with the stress concentration dictates when slip occurs at each point and, as a result, the rupture speed. Thus, the rup- 

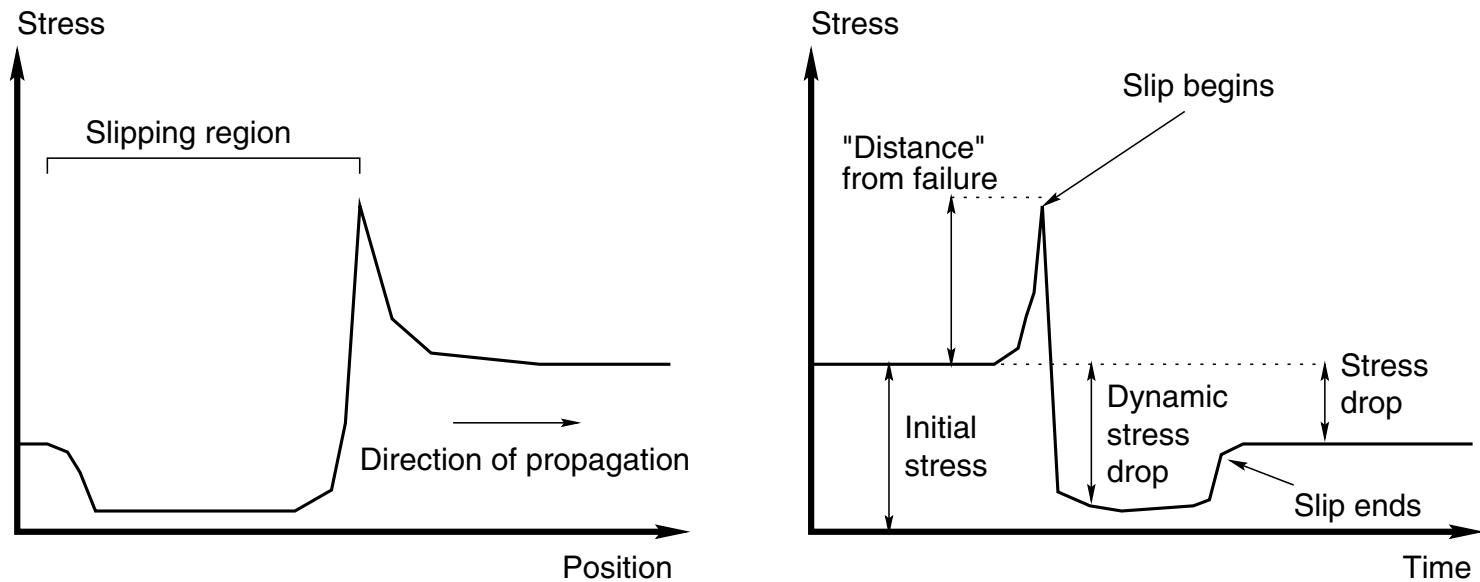

Figure 1. Diagrams of the concentration of shear stress near the rupture front at a specific time as a function of space (left diagram) and at a fixed location as a function of time (right diagram)

ture speed and slip rate are not independent but are related through the dynamic stress drop.

We may also consider the dynamics of the rupture using energy. As the rupture propagates, the rupture front consumes energy through sliding. We associate two forms of energy with the sliding. We call the energy dissipated when the friction decreases during sliding the fracture energy (illustrated in Fig. 2) because it corresponds to the fracture energy in crack models (Rice, 1983). We associate the energy dissipated through sliding at a relatively constant friction stress with the generation of heat. The sliding also releases the energy radiated in the seismic waves. As we increase the fracture energy for a given maximum dynamic stress drop, the rupture consumes more energy leaving less available for radiation. In such cases the slip rates and rupture speed decrease (Rice, 1983; Fukuyama and Madariaga, 1998). Likewise, with a decrease in the fracture energy, more energy is available for sliding, and the slip rates and rupture speed increase. If the fracturing dissipates more energy than the energy released, then the rupture slows and eventually stops. The rupture will also stop propagating if the leading edge of the rupture slows down and it is caught from behind by the trailing edge of the rupture.

Conceptually, we wish to separate the energy dissipated on the fault surface into fracture energy and heat generated by the frictional sliding. However, most conventional friction models define a slip-weakening distance $D_{0}$, where the shear stress decreases from the failure stress to the frictional sliding stress over the slip distance $D_{0}$. If the shear stress decreases linearly over the slip-weakening distance, the fracture energy per unit area equals one-half of the strength excess times the slip-weakening distance (see Fig. 2). In discrete models the slip-weakening distance cannot become arbitrarily small because of the finite discretization size.

Figure 2 shows that the failure stress does not uniquely determine the fracture energy: adjusting the slope of the friction model changes the fracture energy. For example, at a

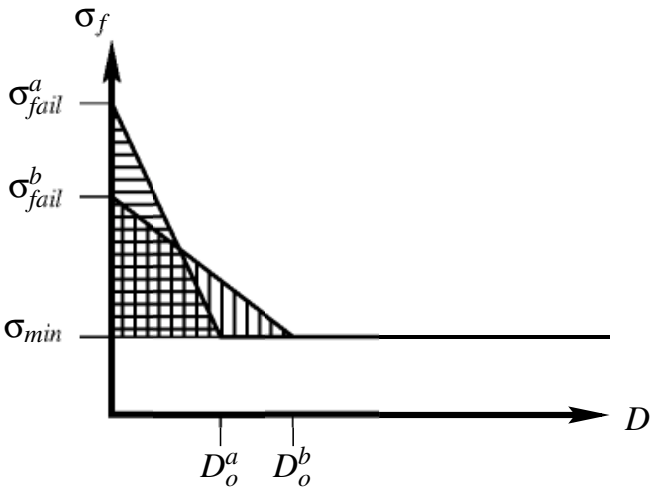

Figure 2. Friction stress $\left(\sigma_{\mathrm{f}}\right)$ as a function of slip distance $(D)$ with an illustration of two sets of parameters for the slip-weakening friction model (denoted by the superscripts a and b) that have the same fracture energy (hatched areas), but different failures stresses $\left(\sigma_{\text {fail }}^{\mathrm{a}}\right.$ and $\left.\sigma_{\text {fail }}^{\mathrm{b}}\right)$ and slip distances $\left(D_{0}^{\mathrm{a}}\right.$ and $\left.D_{0}^{\mathrm{b}}\right)$.

lower failure stress, we can maintain the same fracture energy by reducing the rate at which the friction stress decreases with slip. Guatteri and Spudich (2000) demonstrated this method for an event resembling the $M 6.51979$ Imperial Valley, California, earthquake.

This technique plays a critical role in manipulating the dynamics of the rupture in simulations with discretized domains. Accurately capturing the stress concentration in shear stress near the leading edge of the rupture requires much finer discretization than that necessary to model the wave propagation (Day, 1982b; Madariaga et al., 1998) because the failure stress develops only over a very localized region. We wish to capture the general features of such failure without modeling such localized behavior. In a discrete model, such as a finite-element model, the failure stress becomes a parameter dependent on discretization size, but the fracture energy should continue to control the behavior of the rup- 
ture. We can manipulate the friction model to maintain the same fracture energy for different levels of the failure stress by changing the slip distance as demonstrated in Figure 2. Clearly, this technique breaks down when the slip-weakening distance, $D_{0}$, exceeds the actual amount of slip. Additionally, for a given fracture energy, as the slip-weakening distance and discretization size increase, the rupture jumps more easily to super-shear rupture speeds. Despite these difficulties, we can use larger finite elements than those required to accurately capture the stress concentration and allow the wave propagation to control the local element size without significantly altering the behavior of the rupture. In other words, the failure shear stress in our finite-element models, which determines when a point on the fault begins to slip, is actually some measure of the shear stress at failure averaged over the discretization size. Consequently, it does not correspond to the yield stress in a continuum.

\section{Friction Models}

We focus on two models of sliding friction, both of which compute the upper bound on the friction force from the product of the normal force and the coefficient of friction. We do not implement the state- and rate-dependent friction models advocated by several researchers, including Dieterich (1992) and Scholz (1998), because they are observed at slip rates of less than $1 \mathrm{~mm} / \mathrm{sec}$ and are associated with viscoelastic creep behavior (Persson, 1997); slip during earthquakes occurs at rates of the order of meters per second (Heaton, 1990; Somerville et al., 1999). Furthermore, stateand rate-dependent friction models imply high-sliding friction in the presence of large fault normal compressive stresses. Several plausible mechanisms have been suggested to explain why friction varies in both space and time during earthquakes for dynamic reasons, for example, wrinklelike slip pulses associated with a contrast in material properties (Harris and Day, 1997; Ben-Zion and Andrews, 1998; Anooshehpoor and Brune, 1999), acoustic fluidization (Melosh, 1996), normal vibrations (Brune et al., 1993; Tworzydlo and Hamzeh, 1997), and elastohydrodynamic lubrication (Brodsky and Kanamori, 2001). Instead of choosing to model any particular mechanism, we approximate the general features of the behaviors with simple friction models because several of these mechanisms may be combining to change the friction stress during sliding. Thus, we choose to use simple, $a d$ hoc friction models with characteristics such as slip weakening (decrease in friction with the progression of slip) and rate weakening (increase in friction as the slip rate approaches zero) that produce realistic rupture behavior and capture the general features of the more complicated models. Table 1 provides descriptions of the parameters used in the functional forms of the coefficient of friction.

The two end member cases of rupture models include cracklike behavior, where the healing phases emanate from the boundaries of the fault, and pulselike behavior, where healing phases occur spontaneously and trail behind the leading edge of the rupture (Heaton, 1990). In slip-weak- ening friction models that model true cracklike behavior, the sliding friction drops to some level as slip occurs and remains there; no restrengthening occurs even when sliding stops. With no shear restrengthening, the slip tends to overshoot its final value, and slip occurs whenever seismic waves with a nonzero fault tangential component attempt to propagate across the fault. The slip can by reduced to generally only one episode during a rupture by including shear restrengthening, wherein the sliding friction returns to its initial value upon termination of sliding. As we shall see, pulselike behavior, instead of cracklike behavior, develops when we allow a more gradual increase in friction as the slip rate tends toward zero (rate-weakening behavior).

In the slip-weakening friction model the coefficient of friction decreases linearly from a maximum value to a minimum value over a slip distance of $D_{0}$ :

$$
\mu_{\mathrm{f}}= \begin{cases}\mu_{\max } & \dot{D}(t)=0 \\ \mu_{\max }-\left(\mu_{\max }-\mu_{\min }\right) \frac{D(t)}{D_{0}} & D(t) \leq D_{0} \\ \mu_{\min } & D(t)>D_{0} .\end{cases}
$$

This defines the latent heat (fracture energy) generated by fracture for this friction model. We refer to this model as slip-weakening friction because the material exhibits a weakening in shear strength as slip occurs. Figure 3 illustrates how the coefficient of friction decreases from $\mu_{\max }$ to $\mu_{\min }$ over a slip distance of $D_{0}$. When the slip rate returns to

\section{Table 1}

Description of the Variables Involved in the Friction Models

\begin{tabular}{lcc}
\hline Variable & Dimensions & Description \\
\hline$\mu_{f}$ & Dimensionless & Coefficient of friction \\
$\mu_{\max }$ & Dimensionless & Maximum coefficient of friction \\
$\mu_{\min }$ & Dimensionless & Minimum coefficient of friction \\
$D$ & Length & Slip distance \\
$V, \dot{D}$ & Length/time & Slip rate \\
$D_{0}$ & Length & Slip distance constant \\
$V_{0}$ & Length/time & Slip rate constant \\
\hline
\end{tabular}

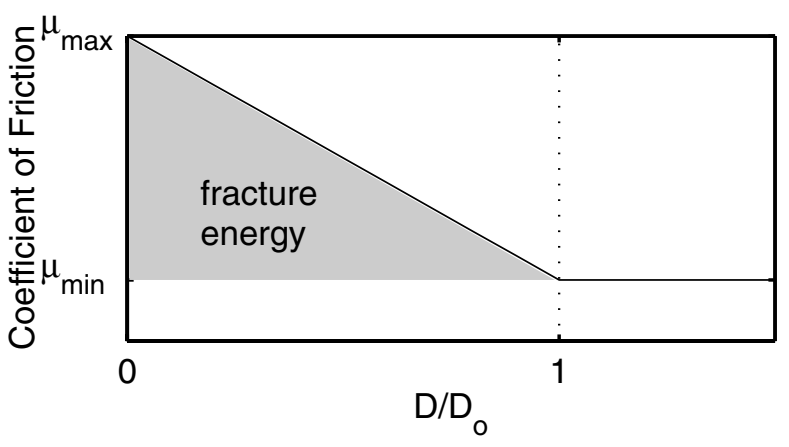

Figure 3. Slip-weakening friction model. The coefficient of friction decreases over the slip distance $D_{0}$. The shaded region is associated with the fracture energy. 
zero, we allow shear restrengthening, so the coefficient of friction returns to $\mu_{\max }$. This results in a smaller static stress drop compared with the dynamic stress drop. Without shear restrengthening the static stress drop may exceed the dynamic stress drop (Madariaga, 1976).

Following Madariaga and Cochard (1996) and Madariaga et al. (1998), we create a second friction model that depends on slip distance and slip rate by taking the greater of the two coefficients of friction determined from the slipweakening friction model and a rate-weakening friction model. As a result, there is no simple expression for the coefficient of friction as a function of slip distance and slip rate. The rate-weakening friction model corresponds to replacing the slip distance in the slip-weakening friction model with the slip rate and the slip distance $D_{0}$ with the slip rate $V_{0}$. We also replace $\mu_{\max }$ in the rate-weakening friction model with $\mu_{\text {post }}$ to allow different shear strengths before and after slip. We refer to this model as slip and rate weakening. Figure 4 illustrates the variation of the coefficient of friction with both slip distance and slip rate, and a typical path during sliding.

\section{Dynamic Energy Balance}

The energy balance provides an additional tool for characterizing an earthquake, and the change in thermal energy allows estimation of the degree of melting on the fault. We derive the dynamic energy balance from the conservation of energy for the entire Earth, assuming no heat is lost on the timescale of the earthquake. We neglect all external forces, such as the gravitational forces from the sun and the other planets, and therefore have no change in the internal energy of the Earth. As given by

$$
E_{\mathrm{R}}+\Delta Q+\Delta W=0
$$

the internal energy of the Earth consists of the radiated energy $\left(E_{\mathrm{R}}\right)$, the change in thermal energy $(\Delta Q)$, and the change in the potential energy $(\Delta W)$. We ignore the rotational energy of the Earth so that the change in potential energy equals the sum of the change in the strain energy and the change in the gravitational potential energy.

When we think about energy and earthquakes, we often only consider the radiated energy because we associate it with the ground motions and can estimate it from groundmotion records. Similarly, in our numerical models the radiated energy is readily available from the earthquake simulation by finding the energy dissipated through the damping matrix.

The primary contribution to the change in the thermal energy comes from the generation of heat by the frictional sliding on the fault. Additionally, the fracturing of materials in the fault zone creates latent heat. The radiated energy eventually dissipates into heat, but we consider it separately as discussed earlier. We include both the fracture behavior and the sliding behavior in the friction model. Consequently, the energy dissipated through frictional sliding includes both

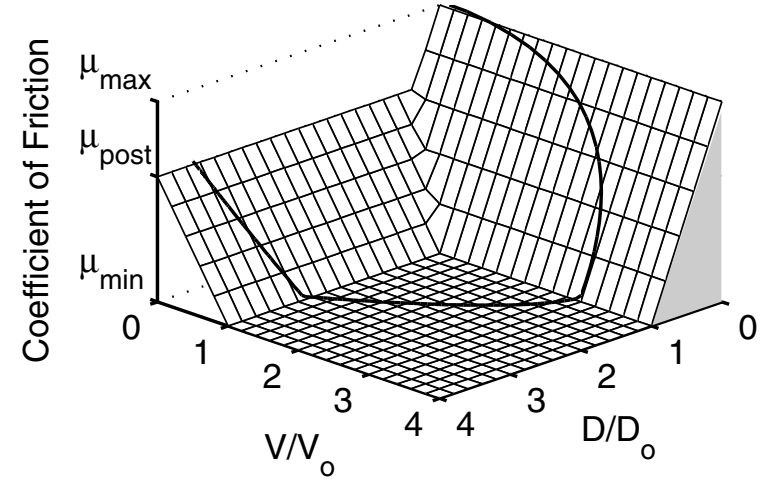

Figure 4. Slip- and rate-weakening friction model. The thick line indicates a typical trajectory of the coefficient of friction. The coefficient of friction decreases over the slip distance $D_{0}$ and then increases when the slip rate drops below $V_{0}$. The shaded region is associated with the fracture energy.

the latent heat associated with the fracture energy and the heat generated by sliding. In order to find the energy dissipated during frictional sliding on the fault $(\Delta Q(t))$, we integrate the increment of heat produced by an increment of slip over the fault surface:

$$
\Delta Q(t)=\iint_{t} \sigma_{\mathrm{f}}(t) \dot{D}(t) \mathrm{d} S \mathrm{~d} t,
$$

where $\sigma_{\mathrm{f}}(t)$ and $\dot{D}(t)$ are the friction stress and slip rate at time $t$.

The heat generated during sliding will increase the temperature in the region surrounding the fault. Because equation (5) includes the fracture energy (which does not induce temperature changes), we use the product of the minimum friction stress during sliding and the final slip to compute the heat per unit area $\left(\Delta Q_{\text {temp }}\right)$ generated by the sliding at each point on the fault. We find the change in temperature at a point on the fault using

$$
\Delta T=\frac{\Delta Q_{\mathrm{temp}}}{C_{\mathrm{v}} \rho d},
$$

where $C_{\mathrm{v}}$ denotes the heat capacity per unit mass, $\rho$ denotes the mass density, and $d$ is the maximum distance perpendicular to the fault to which the heat penetrates.

The distinction between fracture energy and frictional heat is important because only the frictional heat increases the temperature in the sliding zone of the fault. Estimates of the degree of melting in the sliding zone constrain the amount of heat that can be generated over the timescale of the dynamic rupture. On the other hand, fracture energy can be dissipated by anelastic deformation in the vicinity of the leading edge of the propagating rupture (crack tip). Estimates of fracture energy in large earthquakes generally fall in the range of $10^{6} \mathrm{~J} / \mathrm{m}^{2}$, which is 2 to 4 orders of magnitude larger than the fracture energies observed in the laboratory 
(Beroza and Spudich, 1988; Lawn, 1993). Such large fracture energies suggest that anelastic yielding occurs over a much broader region than where the predominant slip takes place.

We define the change in potential energy as the energy released by the slip on the fault, assuming that the slip occurs quasi-statically and that the domain behaves according to linear elasticity. Because both the radiated energy and the change in heat energy must be positive, conservation of energy dictates that the change in potential energy must be negative. This drop in the potential energy allows earthquakes to release energy as propagating waves and to generate heat through frictional sliding.

We follow a procedure similar to that of Dahlen (1977) and Savage and Walsh (1978) to find the change in potential energy caused by an earthquake. Starting with the change in energy for an increment of slip and assuming that the medium behaves linearly elastically gives us

$$
\Delta W=-\frac{1}{2} \int_{S}\left(\sigma_{0}+\sigma_{1}\right) D \mathrm{~d} S,
$$

where $D$ is the slip, and $\sigma_{0}$ and $\sigma_{1}$ are the shear stresses before and after the earthquake, respectively.

From the point of view of understanding the physics of the rupture, we would like to decompose the change in potential energy into change in strain energy and change in gravitational potential energy. As shown by Dahlen (1977) and Savage and Walsh (1978), we cannot determine these changes in energy when we truncate the domain because all points in the Earth contribute terms of the same order in the computations; the domain must encompass the entire Earth in order to compute the change in strain energy or the change in gravitational potential energy. The Appendix contains some additional discussion on how the choice of boundary conditions affects the partitioning of the change in potential energy into changes in strain energy and gravitational potential energy. Therefore, in the energy balance we settle for the total change in potential energy, which is the sum of these two energies.

\section{Dynamic Rupture in a Homogeneous Half-Space}

We first attempt to generate realistic ruptures in a homogeneous half-space. We use some of the basic features of the ruptures observed in nature to judge the behavior of the simulated ruptures. Heaton (1990) and Somerville et al. (1999) examined the rupture behavior of several earthquakes and found no systematic variations in the slip distributions with depth. In other words, the slip distributions could be approximated to first order as the changes in strain on the fault that are independent of depth. In a homogeneous halfspace this corresponds to stress drops that are uniform with depth. For earthquakes in a homogeneous half-space with a uniform stress drop, we expect tapering in the slip along the buried edges of the fault (e.g., see Parsons et al., 1988).
Although difficult to resolve, Heaton (1990) and Somerville et al. (1999) did not find any clear variations in the duration of slip with depth. Consequently, we prefer numerical models where slip rates do not change dramatically with depth. We shall also assume that the nominal tectonic tractions may be derived from the application of relatively uniform strain fields, which is consistent with the absence of any depth dependence found in the distribution of slip from source inversions.

\section{Friction Model Parameters}

We wish to create a relatively uniform slip distribution with depth in a domain where the material properties do not change with depth and the effective fault normal stresses increase linearly with depth as a result of the overburden pressure. For relatively homogeneous slip, the stress drop on the fault will generally vary proportionally with the shear modulus and the slip,

$$
\Delta \sigma=C_{1} \mu D,
$$

where $C_{1}$ is a constant that depends on the rupture dimensions. The stress drop also equals the difference between the initial shear stress, $\sigma_{0}$, and the final shear stress, $\sigma_{1}$. We assume that the initial shear stress comes from a uniform strain field, which gives

$$
\sigma_{0}=\varepsilon_{0} \mu .
$$

For the final shear stress, we use the minimum sliding shear stress,

$$
\sigma_{1}=-\mu_{\min } \sigma_{n}
$$

Combining these equations, substituting in the expression for the shear modulus $\left(\mu=\rho \beta^{2}\right.$, where $\rho$ denotes the mass density and $\beta$ denotes the shear-wave speed), and solving for the minimum coefficient of friction yields

$$
\mu_{\min }=\frac{\left(C_{1} D-\varepsilon_{0}\right) \rho \beta^{2}}{\sigma_{n}} .
$$

We now consider two end cases for the effective normal stresses in our homogeneous half-space: uniform effective normal stresses and lithostatic effective normal stresses. With uniform effective normal stresses, for uniform slip we require a uniform minimum coefficient of friction. This set of parameters, although not physically realistic, is typically used in dynamic rupture simulations (Day, 1982b; Olsen et al., 1997; Madariaga et al., 1998; Harris and Day, 1999; Oglesby et al., 2000b). On the other hand, if the effective normal stress increases linearly with depth, then for uniform slip we need a minimum coefficient of friction that varies inversely with depth. If we try to use a uniform minimum coefficient of friction when the normal stress increases with depth, then the slip will increase rapidly with depth (Aagaard, 1999). Such behavior conflicts with the inferred slip 
distributions from source inversions that exhibit no clear trends with depth (Heaton, 1990; Somerville et al., 1999).

The procedure just described does not yield information regarding the maximum coefficient of friction that we associate with the failure stress and fracture energy. We choose to vary the maximum coefficient of friction with the inverse of the depth as well because this leads to uniform relative changes in the coefficient of friction during sliding. This corresponds to relatively uniform failure stresses, sliding stresses, and fracture energies with depth in the homogeneous half-space.

It is important to note that these constraints on the coefficient of friction arise from our choice to follow the conventional formulation of the friction stress (friction stress equals the product of the coefficient of friction and the normal stress). Alternatively, a more physically meaningful approach might be to assume that the friction force does not depend on the normal stress. For example, the slipweakening friction model can be reformulated to yield the friction stress as a function of slip with low (or zero) sliding friction replacing the minimum coefficient of friction and a drop in friction stress over a given slip distance acting as a proxy for the fracture energy.

\section{Application to Strike-Slip Fault}

We now demonstrate how this parameterization works for the case of dynamic rupture on a strike-slip fault in a homogeneous half-space. The 60-km-long and $15-\mathrm{km}$-wide strike-slip fault lies in a domain $100 \mathrm{~km}$ long, $40 \mathrm{~km}$ wide, and $32 \mathrm{~km}$ deep as shown in Figure 5. The homogeneous half-space has a mass density of $2450 \mathrm{~kg} / \mathrm{m}^{3}$, a shear-wave speed of $3.30 \mathrm{~km} / \mathrm{sec}$, and a dilatational wave speed of 5.70 $\mathrm{km} / \mathrm{sec}$. The finite-element model contains 3.0 million elements and 1.8 million degrees of freedom.
We assume that the effective normal stresses equal the lithostatic pressures. In order to select the initial shear tractions on the fault, we begin by choosing a maximum dynamic stress drop of 2.0 MPa. Recall that because the coefficient of friction returns to its maximum value upon termination of sliding, the dynamic stress drop exceeds the static stress drop. We assume that the earthquake does not completely relieve the initial stresses and apply uniform initial shear tractions of 4.0 $\mathrm{MPa}$ that are tapered at the buried edges of the fault to smother the rupture as it approaches these edges. Figure 6 gives the distributions of the normal and shear tractions on the fault surface.

We also need to determine a value for the failure stress in order to specify the maximum value for the coefficient of friction. We expect the initial stresses to lie somewhere between the minimum sliding shear stresses and the shear stresses at failure. A small distance from failure (the difference between the failure stress and the initial shear stress) implies that the fault is close to failure and that the rupture will propagate very fast. However, note that the choice of failure shear stress depends on the discretization size and that we are actually changing fracture energies. At the other extreme, a large distance from failure inhibits propagation of the rupture. We avoid the extreme cases and select the distance from failure to match the maximum dynamic stress drop. As a result, the initial shear stresses of 4.0 $\mathrm{MPa}$ lie halfway between the minimum sliding shear stresses of 2.0 $\mathrm{MPa}$ and the failure stresses of 6.0 MPa.

Using the normal stresses, we select values for the friction model parameters to yield these choices for the stress drop and the distance from failure. We use the slip-weakening friction model with the two parameters, $\mu_{\max }$ and $\mu_{\min }$, decreasing with the inverse of depth as given by

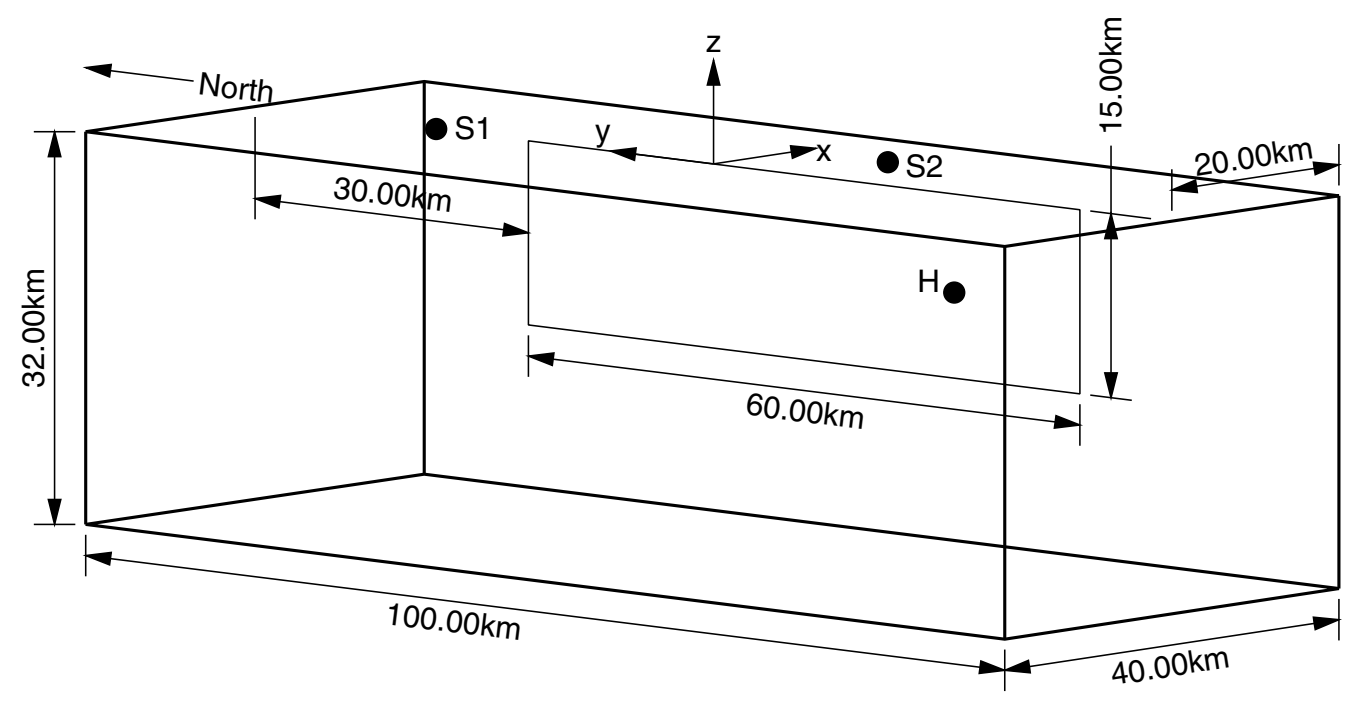

Figure 5. Domain geometry for the strike-slip fault. The label H denotes the hypocenter location. 


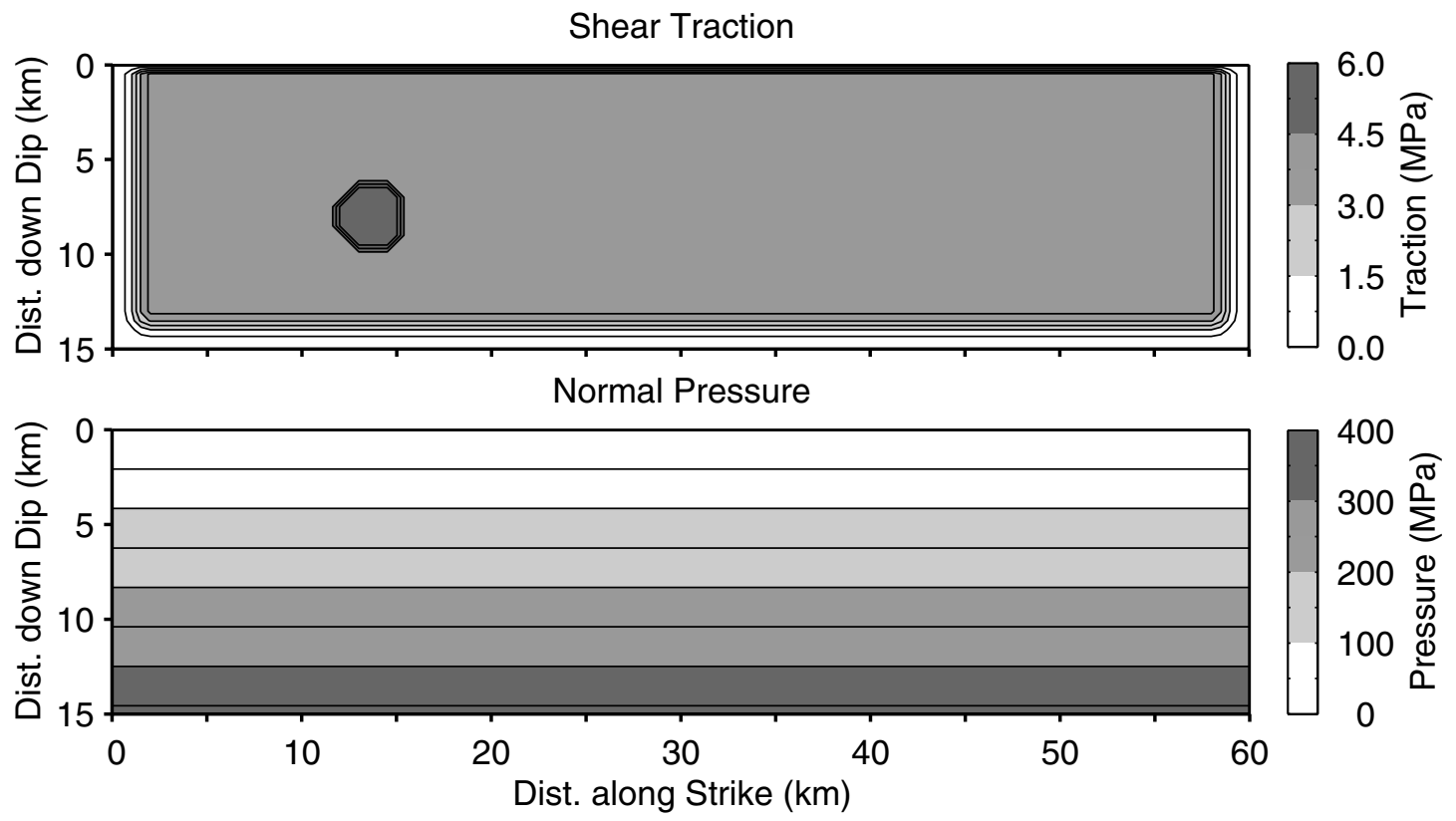

Figure 6. Initial shear tractions and normal pressures on the fault surface of the homogeneous half-space. The shear tractions are derived from a uniform strain field, and the normal pressures increase with depth because of gravity.

$$
\begin{aligned}
& \mu_{\max }= \begin{cases}1.00 & z>-250 \mathrm{~m} \\
\frac{-250 \mathrm{~m}}{z} & z<-250 \mathrm{~m}\end{cases} \\
& \mu_{\min }= \begin{cases}0.333 & z>-250 \mathrm{~m} \\
\frac{-83.3 \mathrm{~m}}{z} & z<-250 \mathrm{~m}\end{cases} \\
& D_{0}=0.150 \mathrm{~m} .
\end{aligned}
$$

It seems unreasonable to let the coefficient of friction approach infinity at the surface, so we clip its value above a depth of $250 \mathrm{~m}$. The slip-weakening distance of $0.150 \mathrm{~m}$ corresponds to a fracture energy $\left(3 \times 10^{5} \mathrm{~J} / \mathrm{m}^{2}\right)$ that yields reasonable rupture speeds.

The snapshots of slip rate in Figure 7 illustrate several important features of the rupture. The rupture expands as an ellipse with a faster rupture speed in the direction of slip compared to the direction perpendicular to slip. In the direction of slip, the rupture displays mode-II crack behavior (shearing), and in the direction perpendicular to slip, the rupture displays mode-III crack behavior (tearing). In the absence of fracture energy, mode-II cracks propagate at the Rayleigh-wave speed, and mode-III cracks propagate at the shear-wave speed (Freund, 1990). However, for reasonable rupture speeds (fracture energies), the rupture speed in the direction of slip (mode-II) tends to exceed the speed in the direction perpendicular to slip (mode-III), because of the asymmetry in the shear-wave radiation pattern (Andrews, 1976b; Day, 1982b; Madariaga et al., 1998). We observe precisely this type of behavior as illustrated by the solid ellipses in the figure that identify the leading edge of the rupture; in the direction parallel to the slip we observe a rupture speed of $2.2 \mathrm{~km} / \mathrm{sec}$ (compared with a shear-wave speed of $3.3 \mathrm{~km} / \mathrm{sec}$ ), and in the direction perpendicular to the slip we observe a rupture speed of $1.8 \mathrm{~km} / \mathrm{sec}$.

When the rupture reflects off the free surface, we fold over the solid ellipse at $5.5 \mathrm{sec}$ to coincide with the reflected portion of the rupture. We also add a dashed ellipse at the leading edge of another portion that propagates along the free surface at a speed of $4.4 \mathrm{~km} / \mathrm{sec}$. This speed lies between the shear-wave speed of $3.3 \mathrm{~km} / \mathrm{sec}$ and the dilatationalwave speed of $5.7 \mathrm{~km} / \mathrm{sec}$. The rupture at the free surface begins to separate at $8.5 \mathrm{sec}$, and at $10 \mathrm{sec}$ two distinct slip events occur at the free surface. We observe substantially larger slip rates where the two portions of the rupture constructively interfere (identified by the intersection of the solid and dashed ellipses). In general, the portion of the rupture traveling faster than the shear-wave speed is associated with larger slip rates than the portion traveling below the shear-wave speed. Furthermore, as the two portions interact, the speed of the portion that propagates below the shearwave speed increases to around $3.0 \mathrm{~km} / \mathrm{sec}$. The solid ellipse that we overlay on the slip rate snapshots reflects this change in rupture speed.

This complex rupture yields an average slip of $1.0 \mathrm{~m}$ and creates the smooth distribution of final slip shown in Figure 8, which is what we expect based on the uniform maximum dynamic stress drop. In other words, beyond the tapering along the edges of the fault, the slip distribution does not exhibit any clear trends with depth, so it is compatible with those from source inversions. In contrast with the final slip, the peak slip rate reflects the complex nature of the rup- 


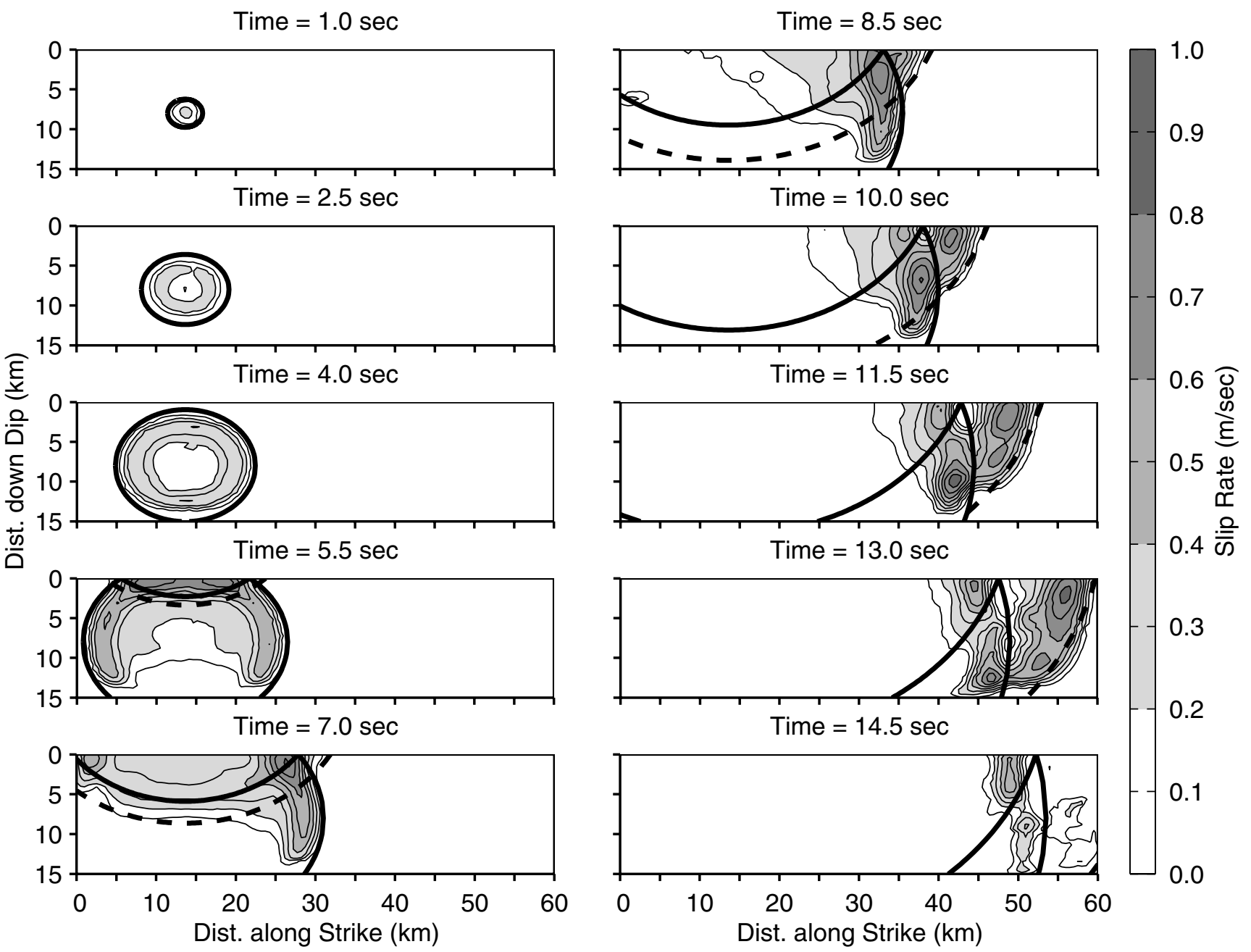

Figure 7. Snapshots of slip rate on the fault surface in the homogeneous half-space with the slip-weakening friction model. The solid and dashed ellipses indicate the leading edges of the ruptures propagating slightly below the shear-wave speed, and between the shear- and dilatational-wave speeds, respectively.

ture. The path of constructive interference between the two portions of the rupture is clearly visible, with slip rates roughly $0.2 \mathrm{~m} / \mathrm{sec}$ greater than in the surrounding regions. We also see large slip rates near the top of the fault at the north end, which come from the faster portion of the rupture.

The faster portion of the rupture propagates at a speed between the shear-wave speed and the dilatational-wave speed, whereas the slower portion propagates at a speed below the shear-wave speed. Both Burridge et al. (1979) and Freund (1979) found steady-state solutions for propagation at speeds both slower than the Rayleigh-wave speed and between the shear-wave speed and the dilatational-wave speed. They concluded that stable propagation can occur for mode-II cracks with speeds of $\sqrt{2}$ times the shear-wave speed. Furthermore, Rosakis et al. (1999) observed cracks propagating at $\sqrt{2}$ times the shear-wave speed in a brittle polyester resin under far-field loading. In our simulation, the faster portion of the rupture propagates at approximately 1.3 times the shear-wave speed or within $6 \%$ of $\sqrt{2}$ times the shear-wave speed.

The super-shear rupture speeds in our simulation arise from the large slip rates along the ground surface. When the rupture hits the free surface, it encounters a reduced resistance to slip. With a hypocenter several kilometers below the surface, a portion of the rupture front several kilometers long hits the ground surface nearly simultaneously and creates a high apparent velocity along the ground surface. Consequently, the slip rates increase and the rupture sustains a super-shear propagation speed along the surface. On a long fault (as in our strike-slip fault above) this leads to bifurcation of the rupture into a portion propagating at approximately $\sqrt{2}$ times the shear-wave speed and a portion propagating a little below the shear-wave speed.

Whereas the portion propagating faster than the shearwave speed appears feasible, source inversions of earthquakes indicate that the ruptures generally propagate at 
Final Slip
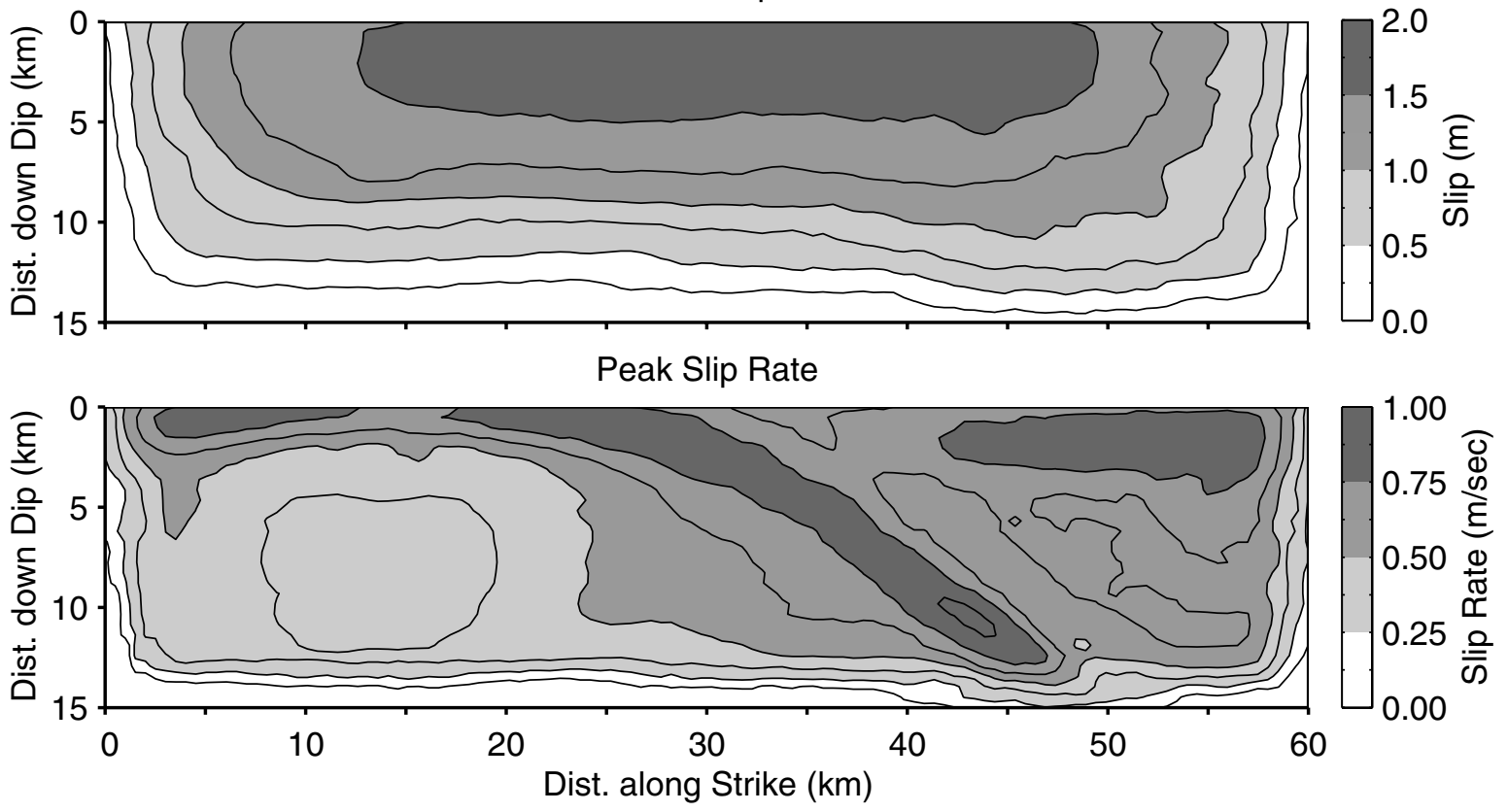

Figure 8. Distributions of final slip and peak slip rate on the fault surface in the homogeneous half-space. The bifurcation of the rupture generates complexity in the distribution of peak slip rate but has little effect on the distribution of final slip.

speeds below the shear-wave speed (Heaton, 1990; Somerville et al., 1999), although some evidence exists for supershear rupture speeds over small portions of fault ruptures (Archuleta, 1984; Hernandez et al., 1999). This discrepancy in the speed of propagation may be explained by two shortcomings of the numerical simulations of ruptures in a discretized, homogeneous half-space. A finer discretization size allows a larger failure stress for the same fracture energy. This tends to localize the stress concentration and to inhibit the transition to super-shear rupture speeds. Higher fracture energies would also impede the transition to supershear rupture speeds. Additionally, the Earth includes variations in the material properties with generally softer material at shallow depths. This reduces the initial stress on the fault for a given amount of strain and reduces the rupture speed as it propagates toward the surface. Hence, the use of too small a fracture energy for the homogeneous material properties may be responsible for the discrepancy in the rupture speeds between the simulation and the real earthquakes.

\section{Dynamic Rupture in a Layered Half-Space}

Having created a dynamic rupture on a strike-slip fault in a homogeneous half-space that exhibits behavior generally compatible with source inversions, we now study dynamic rupture on faults in a layered half-space. Additionally, we attempt to match the general characteristics of the dynamic ruptures with those of the prescribed ruptures from our previous study of near-source ground motions (Aagaard, 1999; Aagaard et al., 2001).
We consider both a vertical strike-slip fault and a shallow-dipping thrust fault. The geometry of the strike-slip domain matches that of the strike-slip fault in the homogeneous half-space discussed earlier and shown in Figure 5. The geometry of the thrust fault resembles the geometry of the Elysian Park fault underneath Los Angeles (Hall et al., 1995). The $28-\mathrm{km}$-long and $18-\mathrm{km}$-wide thrust fault dips $23^{\circ}$ to the north. Figure 9 shows the geometry of the thrust fault with the top of the fault sitting $8.0 \mathrm{~km}$ below the ground surface. The domains for both faults contain the same variation of the material properties with depth. Figure 10 gives the mass density, shear-wave speed, and dilatational-wave speed as a function of depth in the layered half-space. We partition the finite-element meshes among 16 processors using the METIS library (Karypis et al., 1999) from the University of Minnesota. The strike-slip fault simulations required $5.6 \mathrm{hr}$ and the thrust fault simulations required $2.6 \mathrm{hr}$ on the Hewlett-Packard X-Class supercomputer at the Center for Advanced Computing Research at Caltech.

\section{Friction Model Parameters}

We return to equation (11),

$$
\mu_{\min }=\frac{\left(C_{1} D-\varepsilon_{0}\right) \rho \beta^{2}}{\sigma_{n}},
$$

with the objective of creating slip distributions that do not vary systematically with depth. In contrast with the homogeneous half-space, the mass density and shear-wave speed follow a complex variation with depth in the layered half- 


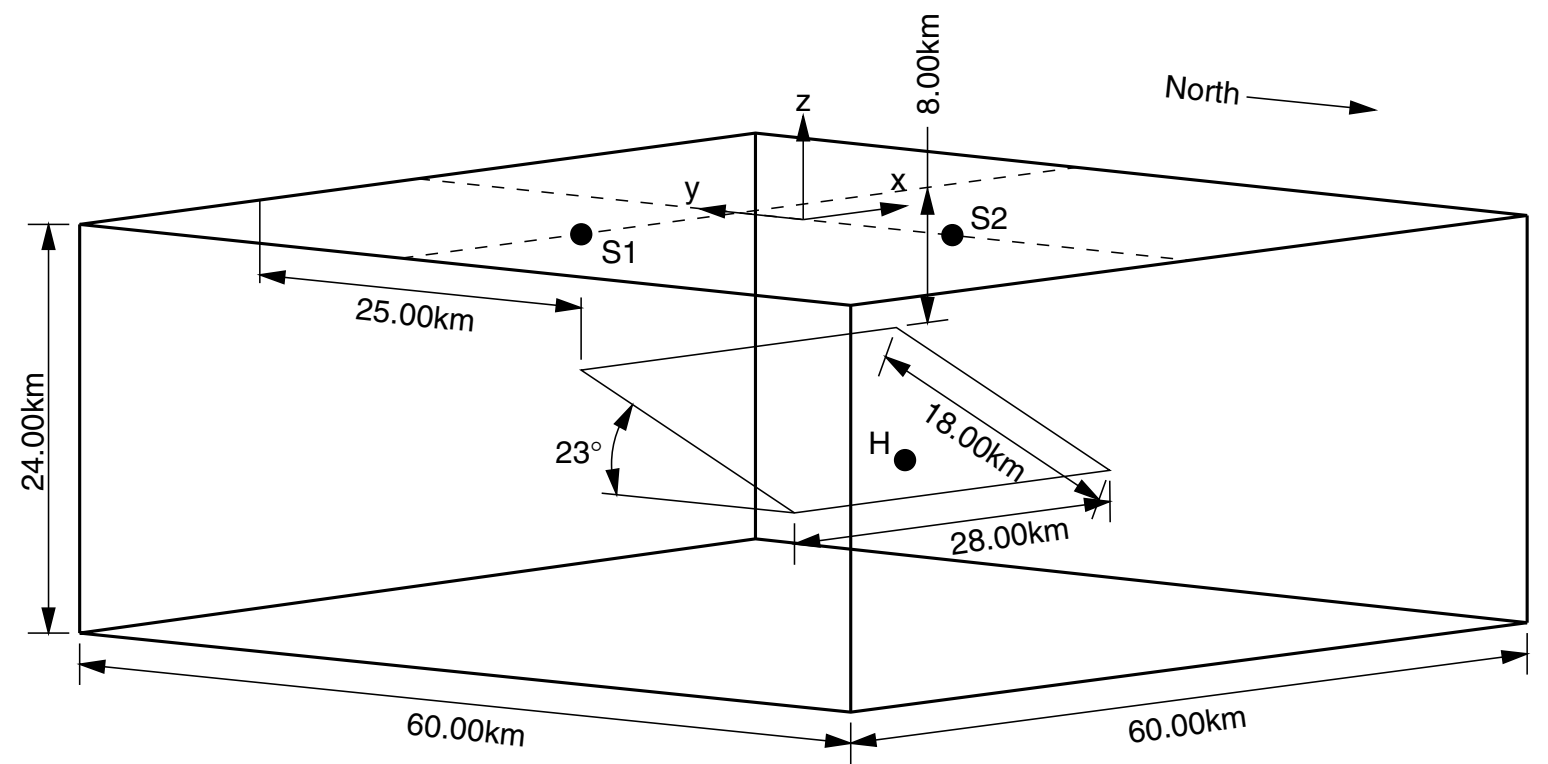

Figure 9. Domain geometry for the thrust fault for the case where the top of the fault lies $8.0 \mathrm{~km}$ below the ground surface. The label $\mathrm{H}$ denotes the hypocenter location.

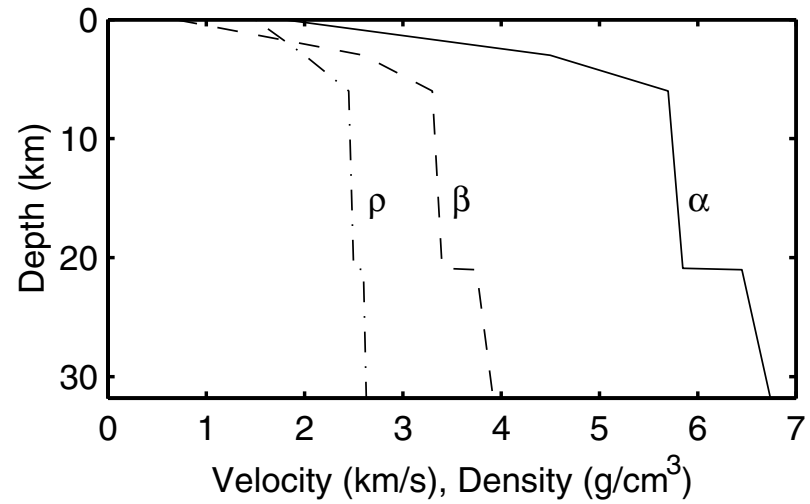

Figure 10. Density $(\rho)$, shear-wave speed $(\beta)$, and dilatational-wave speed $(\alpha)$ as a function of depth in the layered half-space.

space. Above a depth of $6.0 \mathrm{~km}$, the values increase with depth piecewise linearly, whereas below a depth of $6.0 \mathrm{~km}$ the material properties are relatively uniform. Making the coefficient of friction proportional to either the ratio of the square root of the shear modulus to the depth or the ratio of the shear-wave speed to the depth provides a reasonable match to the desired variation of $\mu_{\min }$ given by equation (13), with the material properties in the layered half-space. We choose to vary the minimum coefficient of friction with the ratio of the square root of the shear modulus to the depth. As noted earlier in our discussion of the friction model parameters for the homogeneous half-space, formulating the friction stress from the product of the coefficient of friction and the normal stress in the presence of lithostatic normal pressures requires variations in the coefficient of friction over the depth of the fault.

\section{Simulation Nomenclature}

We name each scenario based on the choice of simulation parameters. Table 2 displays the correspondence between the letters and numbers of the scenario names and the simulation parameters.

\section{Application to Strike-Slip Fault}

We follow the same procedures that we used for the dynamic rupture simulations in the homogeneous half-space to determine the initial shear and normal tractions on the fault surface in the layered half-space. For each scenario, we assume that the pore pressures are negligible and apply lithostatic effective normal tractions. We aim for an average slip of $2.0 \mathrm{~m}$. The average final stress drop on a rectangular, vertical strike-slip fault in a homogeneous Poissonian halfspace approximately follows $\Delta \bar{\sigma}=C \mu(\bar{D} / w)$, where

$$
\begin{gathered}
C \approx \begin{cases}C_{D} & w=l \\
C_{D}+0.9[1-(l / w)] & w<l<2 w \\
C_{D}-0.9 & l>2 w\end{cases} \\
C_{D} \approx \begin{cases}1.6 & \text { for surface rupture } \\
2.1 & \text { for deeply buried faults },\end{cases}
\end{gathered}
$$

where $l$ and $w$ denote the length and width of the fault (Heaton et al., 1986). Below a depth of $6.0 \mathrm{~km}$, the material properties on the fault surface are nearly uniform, so we use the shear modulus from a depth of $6.0 \mathrm{~km}$ in equation (14). Applying this equation with an average slip of $2.0 \mathrm{~m}$ and our fault dimensions yields an average stress drop of 2.5 MPa. The recovery of the coefficient of friction upon termination of sliding means that the maximum dynamic stress drop will exceed the average final stress drop. Consequently, 
Table 2

Description of Letters and Numbers Used to Compose the Scenario Names*

\begin{tabular}{lccc}
\hline Fault Type & Fault Depth & / Friction Model & / Heterogeneity \\
\hline SS (strike slip) & $0(0 \mathrm{~km})$ & SW (slip weakening) & U (uniform) \\
TH (thrust) & $8(8 \mathrm{~km})$ & SRW (slip and rate weakening) & HS (heterogeneous stress/strain) \\
& & P (prescribed rupture) & HF (heterogeneous friction) \\
\hline
\end{tabular}

*For example, scenario SS0/SW/U refers to a strike-slip scenario with the top of the fault at a depth of 0 $\mathrm{km}$, a slip-weakening friction model, uniform initial shear tractions, and uniform coefficients in the expression for the coefficient of friction

on the basis of a test simulation with a homogeneous halfspace, we impose a maximum dynamic stress drop of 4.5 MPa below a depth of $6.0 \mathrm{~km}$, where the material properties are relatively uniform.

We assume that some residual shear stresses remain on the fault after the earthquake and that the initial tectonic shear tractions come from a relatively uniform strain field, so we select tectonic shear strains that produce shear tractions of approximately $6.0 \mathrm{MPa}$ at depths where the material properties are nearly uniform. In order to prevent the effective normal stress on the fault surface from vanishing at the ground surface, we also apply uniform axial tectonic strains in the east-west direction (parallel to the $x$ axis). The tectonic strain field (denoted by the superscript $t$ ) is given by

$$
\begin{aligned}
& \varepsilon_{x x}^{t}=-2.93 \times 10^{-5} \\
& \varepsilon_{y y}^{t}=\varepsilon_{z z}^{t}=-v \varepsilon_{x x}^{t} \\
& \varepsilon_{x y}^{t}=1.10 \times 10^{-4} \\
& \varepsilon_{y z}^{t}=\varepsilon_{x z}^{t}=0,
\end{aligned}
$$

where $v$ denotes Poisson's ratio. We superimpose this strain field on the strains associated with the lithostatic tractions generated by gravity (denoted by the superscript $g$ ),

$$
\begin{aligned}
& \varepsilon_{x x}^{g}=\varepsilon_{y y}^{g}=\varepsilon_{z z}^{g}=\frac{1}{3 \lambda+2 \mu} \int_{0}^{z} \rho(s) g \mathrm{~d} s \\
& \varepsilon_{x y}^{g}=\varepsilon_{y z}^{g}=\varepsilon_{x z}^{g}=0,
\end{aligned}
$$

to generate the shear and normal tractions that we apply on the fault surface (Fig. 11).

Having chosen to vary the minimum coefficient of friction with the square root of the shear modulus and the inverse of the depth, we apply the same functional form to the maximum coefficient of friction. This creates a uniform relative change in the coefficient of friction with depth that we also used in the homogeneous half-space. With the variations of the material properties with depth, we cannot match the distance from failure to the maximum dynamic stress drop over the entire depth of the fault as we did in the homogeneous half-space. As a result, we choose to match (in an average sense) the distance from failure with the maximum dynamic stress drop over the depth range of 6.0-15.0 $\mathrm{km}$, where the material properties remain relatively uniform.
The functional forms of the parameters in the slip-weakening friction model are given by

$$
\begin{aligned}
& \mu_{\max }= \begin{cases}0.164 & z>-1.0 \mathrm{~km} \\
-3.02 \times 10^{-3}\left(\frac{\mathrm{m}^{3} \mathrm{sec}^{2}}{\mathrm{~kg}}\right)^{1 / 2} \frac{\sqrt{\mu}}{z} & z<-1.0 \mathrm{~km}\end{cases} \\
& \mu_{\min }= \begin{cases}0.0235 & z>-1.0 \mathrm{~km} \\
-4.31 \times 10^{-4}\left(\frac{\mathrm{m}^{3} \mathrm{sec}^{2}}{\mathrm{~kg}}\right)^{1 / 2} \frac{\sqrt{\mu}}{z} & z<-1.0 \mathrm{~km}\end{cases} \\
& D_{0}=0.338 \mathrm{~m} .
\end{aligned}
$$

We clip the values above a depth of $1.0 \mathrm{~km}$ to prevent them from approaching infinity at the ground surface. Note that $\mu_{\max }$ and $\mu_{\text {min }}$ denote constants in the friction model, whereas $\mu$ denotes rigidity. Figure 12 shows the maximum and minimum coefficients of friction as a function of distance downdip on the fault.

Figure 12 also displays the initial shear stress through the center of the asperity used to start the rupture, the shear stress at failure, and the minimum sliding shear stress over the depth of the fault. The maximum dynamic stress drop (difference between the initial and minimum sliding shear stresses) closely follows the variation of the shear modulus that increases piecewise linearly down to a depth of $6.0 \mathrm{~km}$ and is nearly uniform below $6.0 \mathrm{~km}$; this means that the change in shear strain, and hence of slip, will tend to be relatively uniform. The fracture energy per unit area (Fig. 12) follows the variation of the failure and the minimum sliding shear stresses with depth. Consequently, the fracture energy remains nearly independent of depth below a depth of $6.0 \mathrm{~km}$, but becomes progressively smaller above a depth of $6.0 \mathrm{~km}$. Using the nomenclature given in Table 2, we shall refer to this scenario as $\mathrm{SSO} / \mathrm{SW} / \mathrm{U}$.

The rupture begins propagating at about $2.5 \mathrm{~km} / \mathrm{sec}$ in the direction parallel to slip (mode-II), and after hitting the ground surface it maintains a speed of $3.0 \mathrm{~km} / \mathrm{sec}(91 \%$ of the local shear-wave speed) in the direction parallel to the slip at a depth of $6.0 \mathrm{~km}$. We attribute the change in the rupture speed to the increase in the peak slip rates as the rupture approaches the ground surface and encounters a reduction in the stiffness and fracture energy. The rupture reflects off the ground surface, but this additional slip soon 


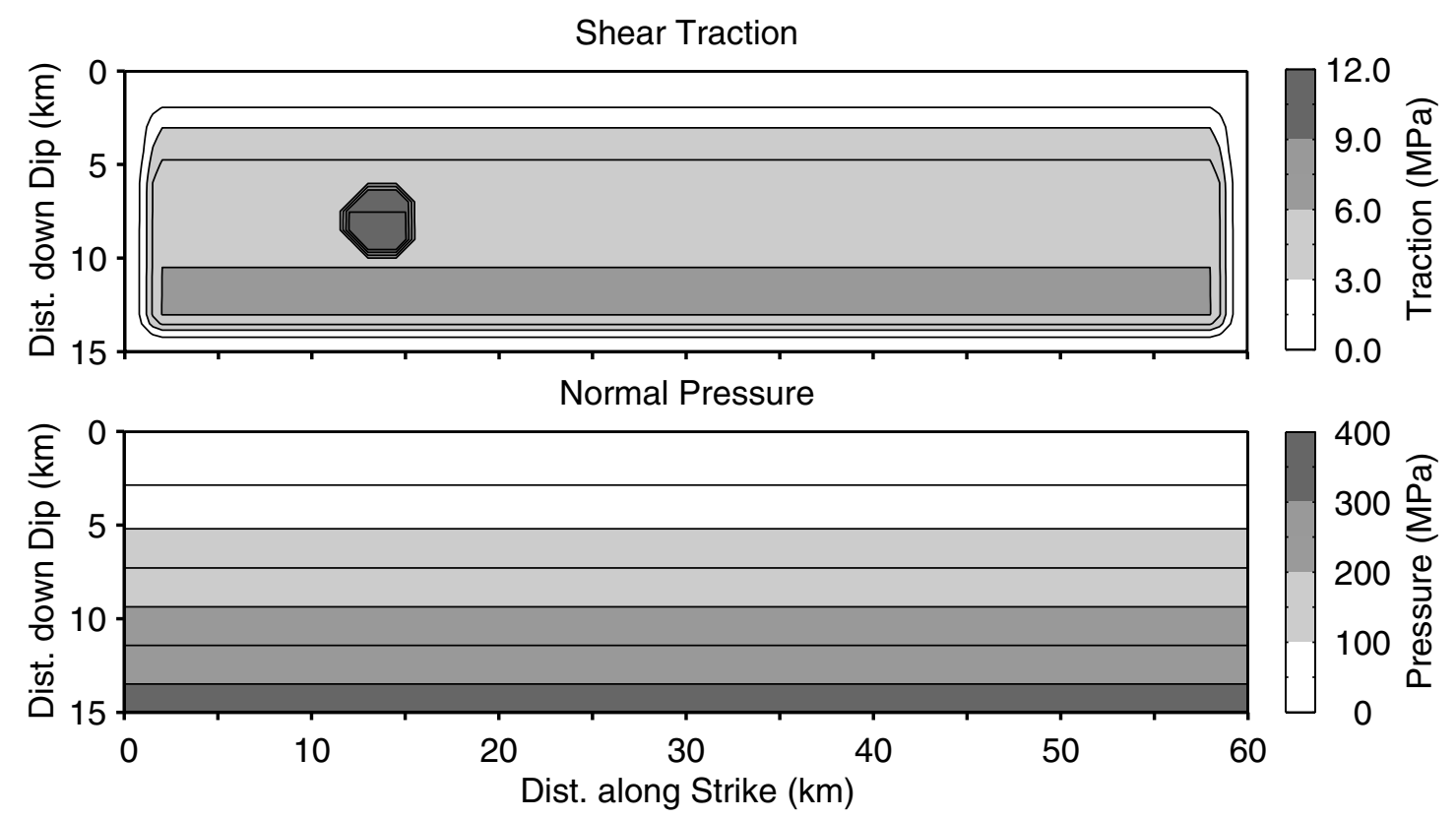

Figure 11. Initial shear tractions and normal pressures on the fault for scenario $\mathrm{SSO} / \mathrm{SW} / \mathrm{U}$. The tractions result from the superposition of the tectonic strains and the strains caused by gravity (equations 15 and 16).
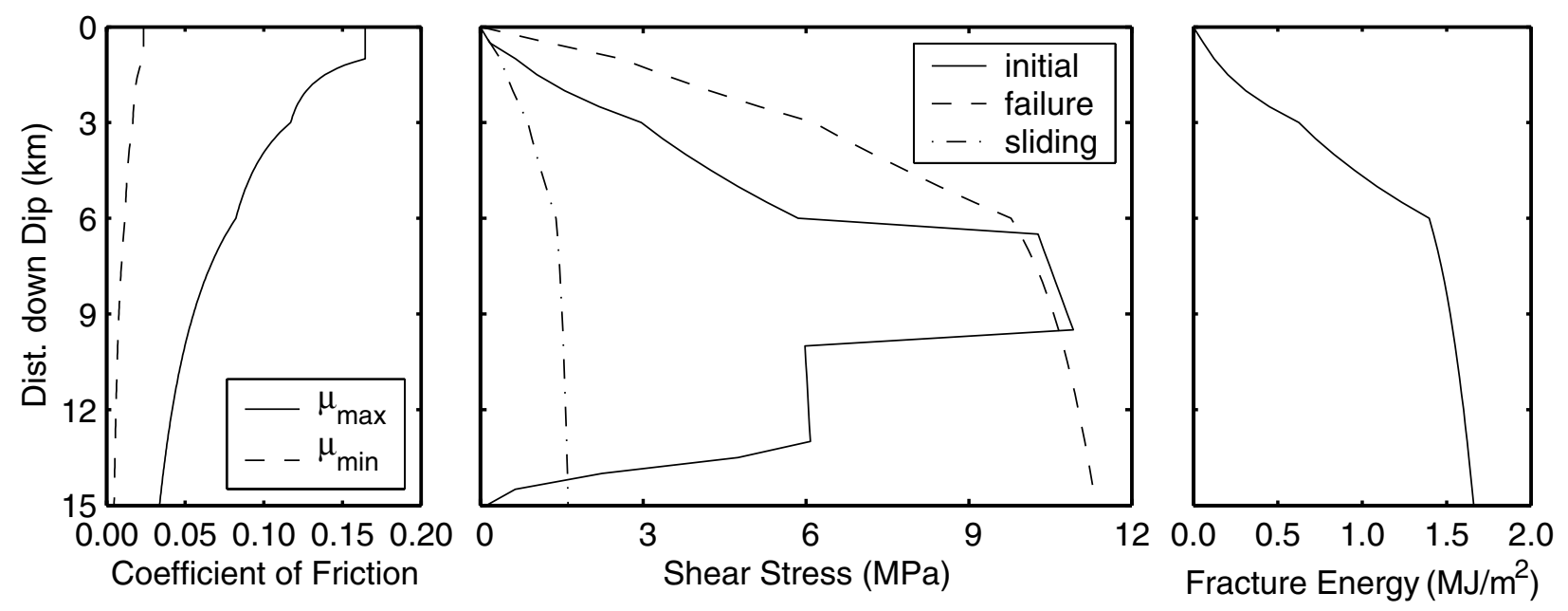

Figure 12. Maximum and minimum coefficients of friction (left), initial, failure, and minimum sliding shear stresses through the center of the asperity (center), and fracture energy per unit area (right) as functions of distance downdip on the fault for scenario SS0/SW/U. The fracture energy per unit area follows the variation of the failure and minimum sliding shear stresses with depth. Whereas we can increase $\mu_{\max }$ significantly without altering the rupture behavior by decreasing the discretization size, increasing $\mu_{\min }$ results in large temperature changes on the fault (see Fig. 15).

disappears. In contrast with the homogeneous half-space simulation, the rupture does not bifurcate and propagates slower than the local shear-wave speed. The average slip of $1.9 \mathrm{~m}$ nearly matches the target value of $2.0 \mathrm{~m}$ and corresponds to a moment magnitude of 6.9. We compute a final stress drop (averaged over the fault surface) of $1.4 \mathrm{MPa}$, which falls short of the $2.5 \mathrm{MPa}$ final, uniform stress drop predicted by equation (14) for a homogeneous half-space.
The presence of the softer material in the top $6.0 \mathrm{~km}$ of the domain reduces the average stress drop compared with that of a homogeneous half-space with the same average slip.

Figure 13 shows the distributions of the final slip and peak slip rate on the fault surface. The region where the final slip exceeds $3.0 \mathrm{~m}$ coincides with the locations subjected to the additional slip associated with the reflection of the rupture off the free surface. We find that the peak slip rates near 

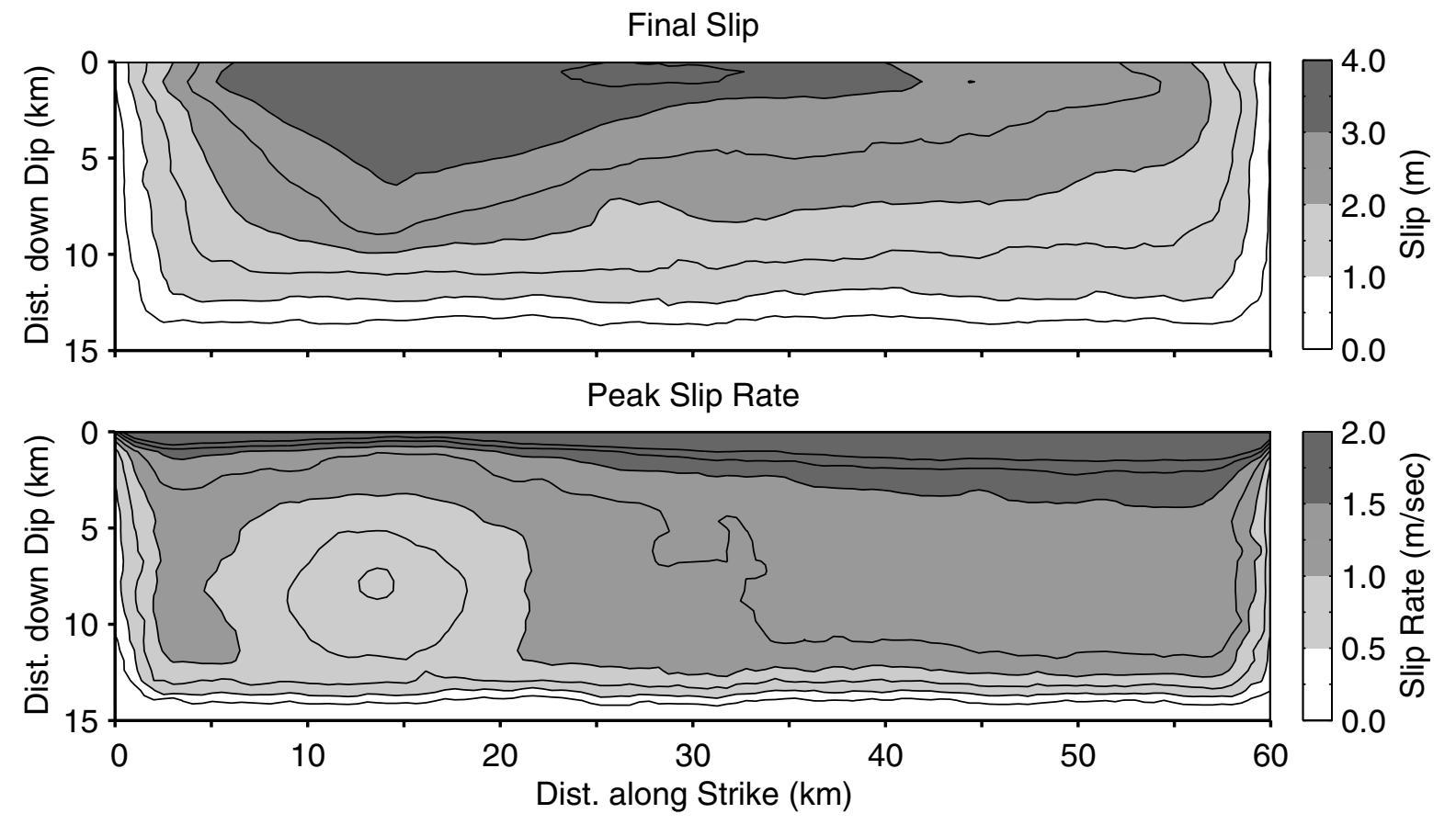

Figure 13. Distributions of final slip and peak slip rate on the fault for scenario $\mathrm{SS} 0 / \mathrm{SW} / \mathrm{U}$. Both the final slip and the peak slip rate are, to first order, independent of depth after removing the tapering along the buried edges of the fault.

the surface are about $0.5 \mathrm{~m} / \mathrm{sec}$ greater than the peak slip rates at depth. The slight tendency for the peak slip rates to increase as the rupture propagates (the peak slip rates increase by roughly $0.25 \mathrm{~m} / \mathrm{sec}$ over a distance of $25 \mathrm{~km}$ ) causes this region of larger slip rates at the surface to progressively increase in size. In contrast with Mikumo (1992), who also used normal stresses equal to the overburden pressure, the slip distributions here do not exhibit a strong variation with depth. This difference stems from the fact that we chose the friction parameters such that the dynamic stress drop follows the change in shear modulus in order to produce relatively uniform changes in strain (slip). Mikumo (1992) decreased the dynamic stress drop with depth following the transition from brittle to ductile behavior of materials, and, as a result, the slip decreased dramatically with depth.

The peak horizontal particle displacements and velocities on the ground surface in Figure 14 clearly illustrate the effect of the rupture directivity on the ground motions. Both the peak horizontal displacements and the velocities increase along the strike of the fault away from the epicenter until the end of the fault where they begin to decay. The peak displacements exceed $1.0 \mathrm{~m}$ over an area of approximately $1200 \mathrm{~km}^{2}$ with a maximum value of $3.0 \mathrm{~m}$. Likewise, the peak velocities exceed $1.0 \mathrm{~m} / \mathrm{sec}$ over an area of approximately $550 \mathrm{~km}^{2}$ with a maximum value of $3.5 \mathrm{~m} / \mathrm{sec}$. Although the peak displacements decay away from the fault at a slower rate than the peak velocities, the most severe motion is confined to a narrow region along the fault. These near- source ground motions display the same principal features as those of Olsen and Archuleta (1996) and Olsen et al. (1997) for similar-sized events on strike-slip faults.

We now evaluate the level of the shear stresses on the fault during sliding. Recall that in most instances adding a constant value to the initial, failure, and sliding shear stresses does not significantly change the rupture behavior (Guatteri and Spudich, 1998). This means that the observed rupture behavior and ground motions do not constrain the absolute levels of these stresses. However, using equation (6) we can compute the change in temperature at each point on the fault. Observations of exposed fault surfaces indicate that slip in an earthquake likely occurs across a region of less than a few millimeters with little melting of the rocks (Chester and Chester, 1998). This implies that the change in temperature on the fault during sliding remains below the level that would cause melting. This is a variation of the classic heatflow problem on the San Andreas fault (Brune et al., 1969; Lachenbruch, 1980). In our simple analysis, we shall assume that the initial temperature at each point on the fault is small compared with the melting temperature and that changes in temperature of the order of $1000 \mathrm{~K}$ cause melting. Thus, we want our sliding stresses to produce temperature changes less than $1000 \mathrm{~K}$.

We estimate the temperature change on the fault during sliding using equation (6) by following a procedure similar to that of McKenzie and Brune (1972), Richards (1976), and Kanamori et al. (1998). We begin by assuming a heat capacity per unit mass of $1000 \mathrm{~J} / \mathrm{kg} \mathrm{K}$. If the slip occurs across 
an infinitesimally thin zone, then the heat is confined to the thermal penetration depth given by $d=\sqrt{k \tau_{d}}$, where $k$ is the thermal diffusivity and $\tau_{d}$ the timescale of the slip. Assuming that $k=1.35 \times 10^{-6} \mathrm{~m} / \mathrm{sec}^{2}$ and choosing $\tau_{d}=5 \mathrm{sec}$ gives $d=2.6 \mathrm{~mm}$. If the slip is distributed over a zone of finite width, then $d$ would be larger. Consequently, we moderate the value of $d$ given by the thermal penetration depth with an infinitesimally thin slip zone and choose a value of $d=$ $5.0 \mathrm{~mm}$. As shown in Figure 15, at most locations the temperature increases by $200-300 \mathrm{~K}$. We observe smaller changes in temperature near the top of the fault because the sliding stresses are smaller there. Below a depth of $6.0 \mathrm{~km}$ the sliding stresses vary little; therefore, below that depth the change in temperature closely resembles the distribution of final slip. Thus, our sliding stresses seem consistent with the lack of melting observed in fault-zone materials. However, if the minimum coefficient of friction was increased to levels reported from laboratory measurements $\left(\mu_{\min } \approx 0.6\right.$ [Dieterich, 1992]), then the temperature changes at middepth on the fault would be 64 times larger than those shown in Figure 15.

On the basis of this scenario, we find that uniform tectonic strains and a friction model with parameters that vary proportionally to the square root of the shear modulus and inversely with depth produce a realistic rupture: we observe rupture speeds and slip distributions that are compatible with source inversions, the ground motions exhibit the directivity that we expect, and the estimated changes in temperature on the fault surface remain consistent with the limited amount of glassy material observed in fault zones. Moreover, changing the dependence of the coefficient of friction from the square root of the shear modulus to the shear-wave speed also yields nearly identical behavior (Aagaard, 1999). Dynamic ruptures with uniform effective normal stresses dis-

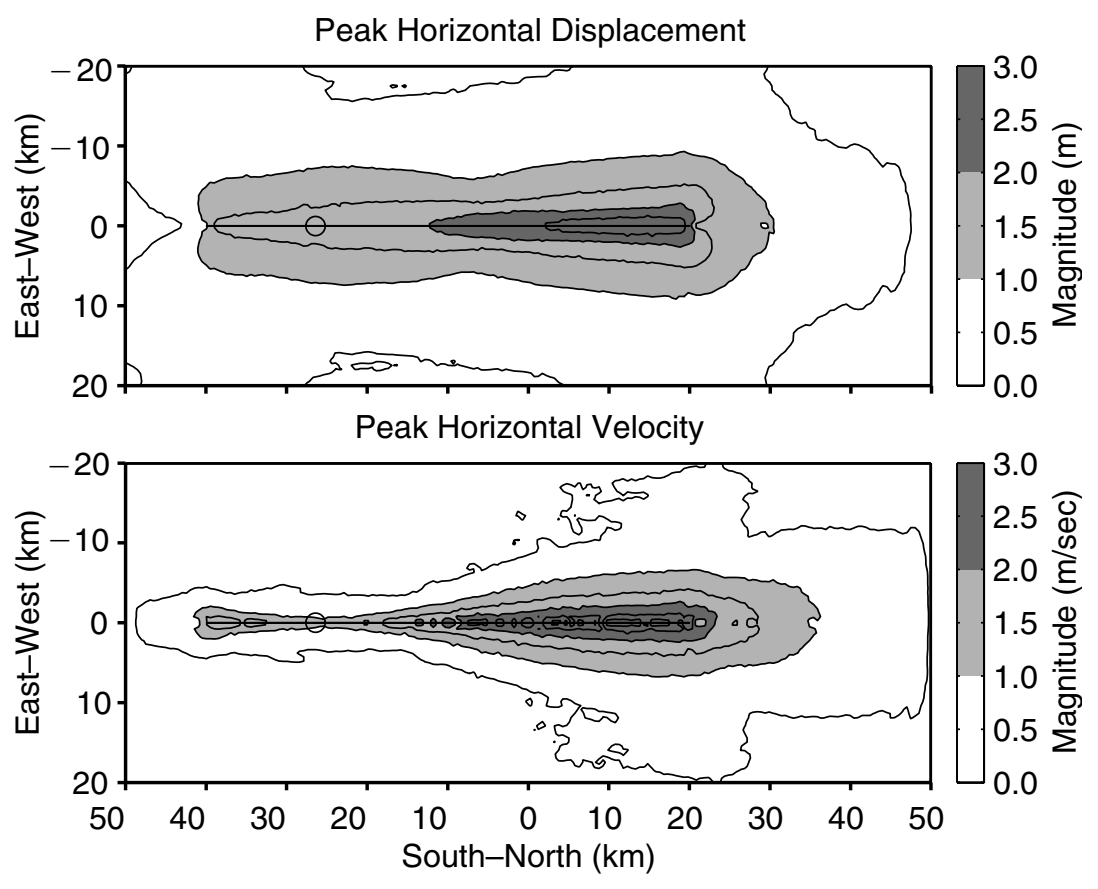

Figure 14. Peak horizontal particle displacements and velocities on the ground surface for scenario SSO/SW/U. The line indicates the projection of the fault plane on to the ground surface, and the hollow circle identifies the epicenter. The amplitude of the ground motions increases along the fault away from the epicenter because of rupture directivity.

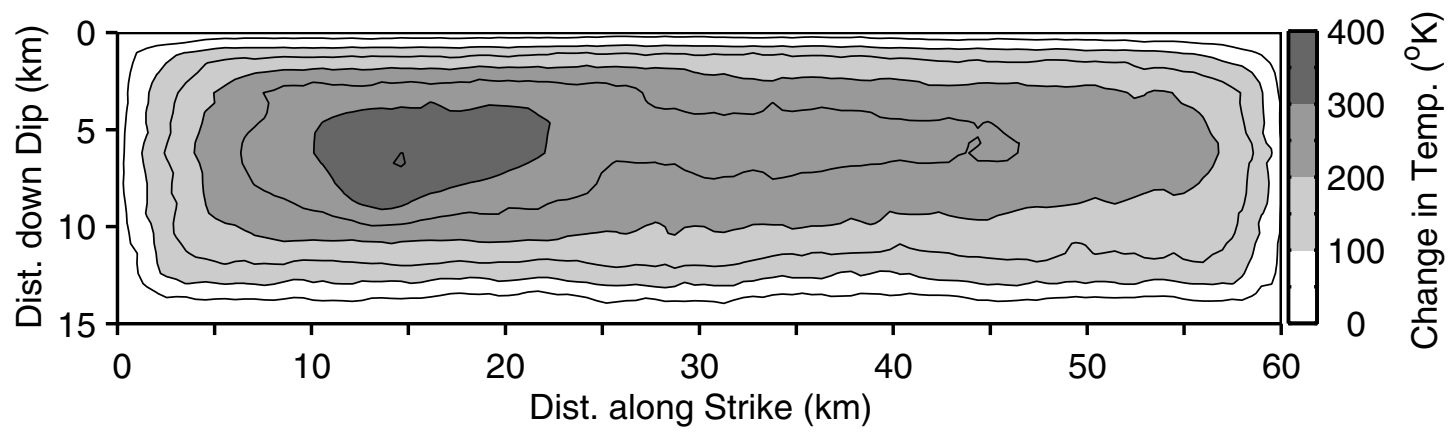

Figure 15. Final change in temperature on the fault for scenario SSO/SW/U. By using very low values of sliding friction (see Fig. 12), the temperature changes are compatible with the lack of melting observed in exposed faults. 
play similar behavior (e.g., Day 1982b; Mikumo and Miyatake, 1993; Madariaga et al., 1998) but would require extremely high pore pressures at depth to be physically meaningful.

\section{Application to Thrust Fault}

We now turn our attention to dynamic ruptures on the thrust fault in the layered half-space (Fig. 9). On dipping faults, the tectonic stresses generate both shear and normal stresses on the fault surface. With pore pressures at or below the hydrostatic pressure, gravity creates effective normal stresses that increase with depth and far exceed the contributions of the tectonic stresses' to the normal stresses. Consequently, changing the dip angle of the fault while keeping the tectonic stresses constant causes almost no variations in the effective normal stresses. This means we can follow the same procedure that we used for the strike-slip fault for dynamic failure on the thrust fault. This would not be the case if we chose to neglect the effect of gravity on the normal stresses and used uniform effective normal stresses.

We apply uniform horizontal axial strains and shear strains to generate the shear tractions on the fault. We align the shear tractions with the desired slip direction, which has a rake angle of $105^{\circ}$ from the strike, and aim for an average slip of $1.0 \mathrm{~m}$. For inclined faults the average stress drop remains proportional to the product of the shear modulus and average slip, but the proportionality constant depends on the depth and dip angle of the fault. Consequently, for inclined faults we do not have a simple expression for the average stress drop as a function of the shear modulus and the average slip that we have for strike-slip faults (equation 14) (Parsons et al., 1988).

With the top of the fault buried $8.0 \mathrm{~km}$ below the ground surface, the material properties exhibit little change over the depth of the fault. As a result, uniform tectonic strains create nearly uniform shear and normal tractions on the fault. The shallow dip of the fault causes the tectonic strains to produce much smaller normal tractions than the normal tractions from gravity. We do not change the functional form of the slip-weakening friction model from the one used in the strike-slip case; the parameters in the friction model continue to depend on the ratio of the square root of the shear modulus to the depth. We do change the coefficients slightly to create the desired maximum dynamic stress drop and shear stresses at failure.

We use homogeneous initial tectonic strains to generate nominal shear tractions of 6.0 MPa on the fault surface and superimpose these tractions on the lithostatic tractions generated by gravity. We again denote the tectonic strains,

$$
\begin{aligned}
& \varepsilon_{y y}^{t}=2.36 \times 10^{-4} \\
& \varepsilon_{x x}^{t}=\varepsilon_{z z}^{t}=-v \varepsilon_{y y}^{t} \\
& \varepsilon_{x y}^{t}=7.27 \times 10^{-5} \\
& \varepsilon_{y z}^{t}=\varepsilon_{x z}^{t}=0,
\end{aligned}
$$

with the superscript $t$ and the strains caused by gravity,

$$
\begin{aligned}
& \varepsilon_{x x}^{g}=\varepsilon_{y y}^{g}=\varepsilon_{z z}^{g}=\frac{1}{3 \lambda+2 \mu} \int_{0}^{z} \rho(s) g \mathrm{~d} s \\
& \varepsilon_{x y}^{g}=\varepsilon_{y z}^{g}=\varepsilon_{x z}^{g}=0
\end{aligned}
$$

with the superscript $g$. We select nominal minimum sliding stresses of 1.5 MPa, a nominal maximum dynamic stress drop of 4.5 MPa, and nominal shear stresses at failure of $10.5 \mathrm{MPa}$, which yields

$$
\begin{aligned}
& \mu_{\max }= \begin{cases}0.162 & z>-1.0 \mathrm{~km} \\
-2.97 \times 10^{-3}\left(\frac{\mathrm{m}^{3} \mathrm{sec}^{2}}{\mathrm{~kg}}\right)^{1 / 2} \frac{\sqrt{\mu}}{z} & z<-1.0 \mathrm{~km}\end{cases} \\
& \mu_{\min }= \begin{cases}0.0231 & z>-1.0 \mathrm{~km} \\
-4.24 \times 10^{-4}\left(\frac{\mathrm{m}^{3} \mathrm{sec}^{2}}{\mathrm{~kg}}\right)^{1 / 2} \frac{\sqrt{\mu}}{z} & z<-1.0 \mathrm{~km}\end{cases} \\
& D_{0}=0.338 \mathrm{~m}
\end{aligned}
$$

for the parameters in the slip-weakening friction model. These match the stresses on the strike-slip fault at similar depths. We will refer to this scenario as TH8/SW/U.

We start the rupture using a shear stress asperity with a radius of $1.8 \mathrm{~km}$ located along the north-south centerline of the fault at a depth of 13.5 or $4.0 \mathrm{~km}$ updip from the bottom of the fault (Fig. 9, hypocenter H). The taper in the shear tractions on all four edges smothers the rupture as it approaches the edges of the fault. Figure 16 displays the initial shear and normal tractions applied to the fault surface.

The rupture begins slowly in response to the placement of the asperity close to the edge of the fault. As the rupture begins to propagate, the rupture front conforms to the familiar elliptic shape with the fastest rupture speed in the direction of slip, which has a rake angle of $105^{\circ}$. The peak slip rates remain relatively low, and the rupture propagates in the direction of slip at a speed of only $2.2 \mathrm{~km} / \mathrm{sec}$ or about $67 \%$ of the local shear-wave speed. The elliptic shape of the rupture front causes the leading edge of the rupture to reach the top of the fault several seconds before the rupture reaches the lateral edges; this gives the rupture a bilateral appearance.

The distribution of final slip displayed in Figure 17 displays no clear trends with depth and resembles the final slip of a statically applied uniform stress drop. The average slip of $1.2 \mathrm{~m}$ agrees reasonably well with the target value of 1.0 $\mathrm{m}$. The reflection of the dilatational wave off the ground surface generates a shear wave that propagates back down toward the fault. As the wave passes through the fault, the dynamic shear stresses cause additional sliding on the fault and the peak slip of $2.3 \mathrm{~m}$ near the hypocenter. The slip rates associated with this additional slip near the hypocenter exceed those in the same region for the first slip event, which reflect the slow initiation of the rupture. If we neglect the 

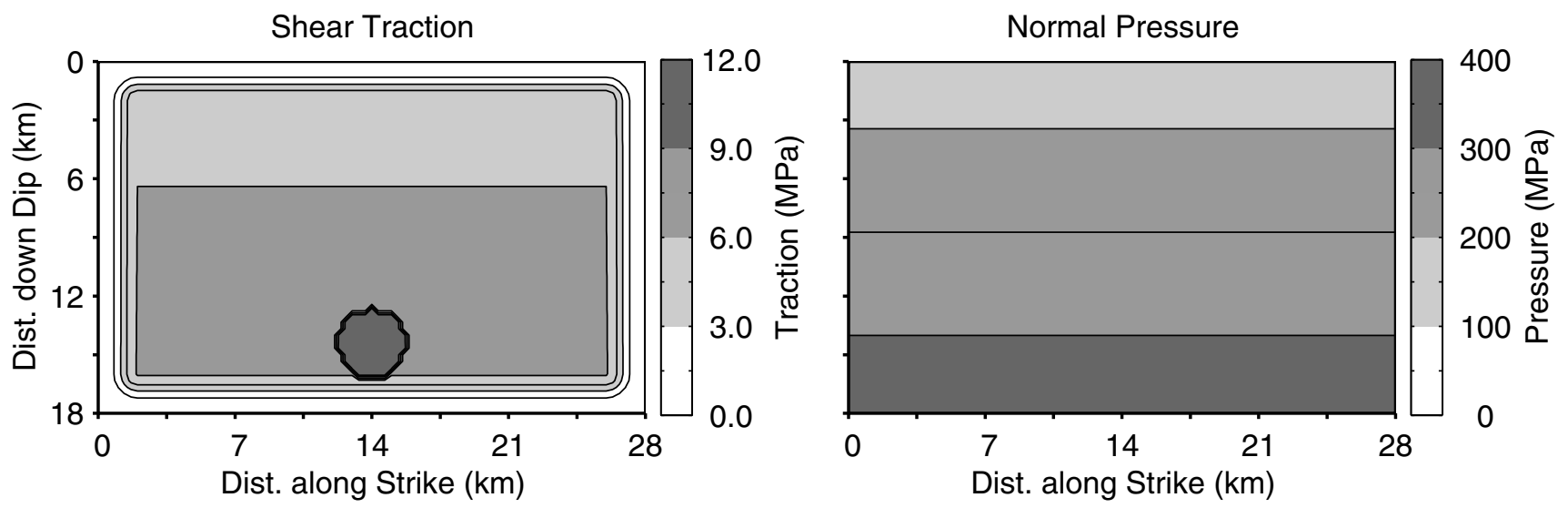

Figure 16. Initial shear tractions and normal pressures on the fault for scenario $\mathrm{TH} 8 / \mathrm{SW} / \mathrm{U}$. The tractions result from the superposition of the tectonic strains and the strains caused by gravity (equations 18 and 19).
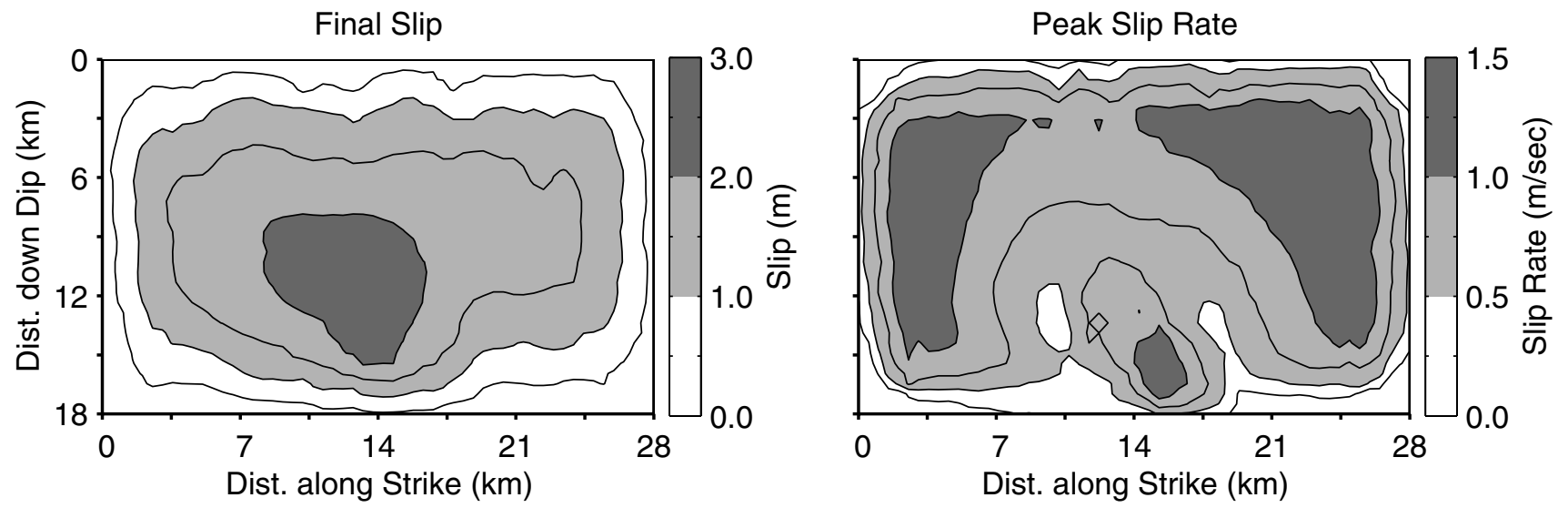

Figure 17. Distributions of final slip and peak slip rate on the fault for scenario TH8/SW/U. Both distributions exhibit no clear depth dependence.

high slip rates near the hypocenter that correspond to the second slip event, then we find that the peak slip rates progressively increase as the rupture propagates.

The relatively slow rupture speed of $67 \%$ of the local shear wave speed allows limited reinforcement of the seismic waves by the rupture. Additionally, the bilateral nature of the end of the earthquake directs energy laterally, instead of updip from the fault. These effects, along with the smaller fault size, result in significantly smaller ground motions than those from the strike-slip fault. The distribution of the peak horizontal displacements and velocities in Figure 18 shows the directivity of the rupture even at this slow rupture speed. The propagation of the rupture toward the surface along the center of the fault creates the local peaks in the horizontal displacements south of the top of the fault. The bilateral nature of the end of the rupture, coupled with the southsoutheast slip direction, creates the large displacements toward the east. We attribute the local peak in the displacements north of the epicenter to constructive interference among the waves coming from the east and west ends of the fault and the second slip event near the hypocenter. See Aagaard (1999) and Aagaard et al. (2001) for a detailed discussion of the differences between the near-source ground motions for the strike-slip fault and the thrust fault used here.

We examine the changes in temperature on the fault surface to determine if melting occurs. As in the case of the strike-slip fault, we assume a heat capacity per unit mass of $1000 \mathrm{~J} / \mathrm{kg} \mathrm{K}$ and confinement of the heat to a region that extends $5.0 \mathrm{~mm}$ perpendicular to the fault. Figure 19 shows that the distribution of the change in temperature closely follows the distribution of final slip. This is caused by the nearly uniform minimum sliding stresses. Over most of the fault the maximum temperature change does not exceed 250 $\mathrm{K}$. Although the temperature change does approach $300 \mathrm{~K}$ in the hypocentral region, the changes in temperature remain consistent with the lack of melting in exposed fault zones.

As expected, the uniform strain field and the friction model with dependence on both the shear modulus and the depth produce a rupture that generally conforms to the behavior of earthquake ruptures. The location of the asperity 

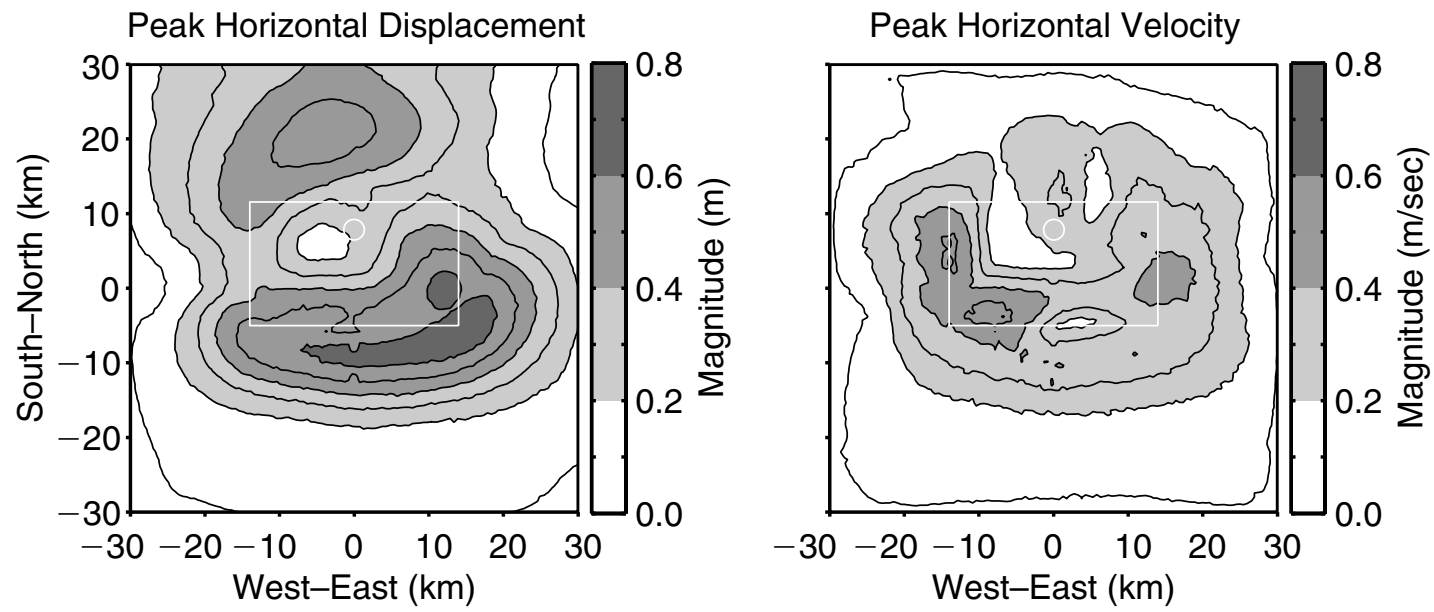

Figure 18. Peak horizontal particle displacements and velocities on the ground surface for scenario TH8/SW/U. The white line indicates the projection of the fault plane on to the ground surface, and the white circle identifies the epicenter. The slow rupture speed of $67 \%$ of the local shear-wave speed limits the directivity of the rupture, which results in small amplitude motions.

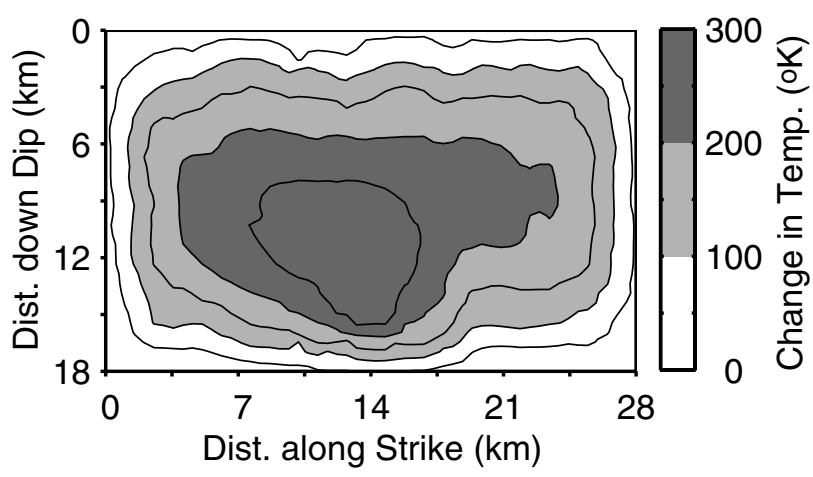

Figure 19. Final change in temperature on the fault for scenario TH8/SW/U. The temperature changes are compatible with the limited amount of melting observed in exposed faults.

used to start the rupture near the edge of the fault slows the initiation of the rupture and leads to a slow rupture speed. Nevertheless, the rupture creates smooth distributions of final slip and peak slip rate that agree with our understanding of uniform stress drop earthquakes. Additionally, the level of sliding stress appears realistic based on the lack of melting associated with the estimated changes in temperature.

\section{Effect of Type of Friction Model}

We study the sensitivity of the ground motions to the inclusion of rate weakening (dependence of the coefficient of friction on slip rate) using two friction models. For the strike-slip fault, we consider the slip-weakening friction model (scenario SS0/SW/U discussed earlier) and the slipand rate-weakening friction model (scenario SS0/SRW/U). Additionally, we compare the same two types of friction models for the top of the thrust fault placed at the ground surface (scenarios TH0/SW/U and TH0/SRW/U).

The minimum sliding stresses remain the same for both strike-slip scenarios, but we increase the maximum dynamic stress drop by a factor of 1.44 for scenario SSO/SRW/U. The slip- and rate-weakening friction model requires a greater dynamic stress drop to generate the same slip as the slipweakening friction model. We also scale the coefficient of friction at failure in order to maintain a distance from failure that matches the maximum dynamic stress drop. The function forms of the parameters in the friction model for scenario $\mathrm{SS} 0 / \mathrm{SRW} / \mathrm{U}$ are given by

$$
\begin{aligned}
& \mu_{\max }= \begin{cases}0.227 \\
-4.17 \times 10^{-3}\left(\frac{\mathrm{m}^{3} \mathrm{sec}^{2}}{\mathrm{~kg}}\right)^{1 / 2} \frac{\sqrt{\mu}}{z} & z<-1.0 \mathrm{~km}\end{cases} \\
& \mu_{\text {min }}= \begin{cases}0.0235 \\
-4.31 \times 10^{-4}\left(\frac{\mathrm{m}^{3} \mathrm{sec}^{2}}{\mathrm{~kg}}\right)^{1 / 2} \frac{\sqrt{\mu}}{z} & z<-1.0 \mathrm{~km}\end{cases} \\
& \mu_{\text {post }}= \begin{cases}0.157 \\
-2.88 \times 10^{-3}\left(\frac{\mathrm{m}^{3} \mathrm{sec}^{2}}{\mathrm{~kg}}\right)^{1 / 2} \frac{\sqrt{\mu}}{z} & z<-1.0 \mathrm{~km}\end{cases} \\
& D_{0}=0.446 \mathrm{~m} \\
& V_{0}=0.150 \mathrm{~m} / \mathrm{sec} .
\end{aligned}
$$

Recall that $V_{0}$ denotes the slip rate at which shear rate weakening occurs in the slip- and rate-weakening friction model (see Fig. 4). The change in the parameters with respect to equation (17) corresponds to the increase in the dynamic stress drop as well as the increase in the fracture energy 
needed to prevent super-shear rupture speeds. These initial conditions for scenario SSO/SRW/U produce an earthquake with a moment magnitude of 6.8 and an average slip of $1.4 \mathrm{~m}$.

In scenario SS0/SRW/U the trailing edge of the rupture follows much closer behind the leading edge of the rupture than in scenario SSO/SW/U. This significantly reduces the region where slip is occurring at any given moment and generates a pulselike rupture compared with the cracklike rupture produced by the slip-weakening friction model. However, because we allow instantaneous recovery of the coefficient upon termination of sliding in the slip-weakening friction model, our slip-weakening friction model creates a departure in the rupture behavior from conventional cracklike ruptures where the maximum dynamic stress drop matches the static stress drop. As a result, for both friction models the vast majority of the slip at each point occurs soon after the initiation of sliding at that point. In scenario SSO/ SRW/U the healing portion of the rupture almost catches the leading edge of the rupture, and this narrowing of the rupture in the central portion of the fault significantly reduces the peak slip rates and nearly smothers the rupture. Fukuyama and Madariaga (1998) and Nielsen and Olsen (2000) also observed narrowing of the rupture when they included rate weakening in a similar friction model.

The velocity time histories at sites S1 and S2 displayed in Figure 20 reflect the weak sensitivity of the ground motions to the friction model. As shown in Figure 5, site S1 lies on the ground surface $10 \mathrm{~km}$ north of the north tip of the fault, and site $\mathrm{S} 2$ lies on the ground surface $10 \mathrm{~km}$ east of the center of the fault. At site S1 we find very little difference in the ground motions for the two friction models. At site S2 where the amplitude of the motion is about onethird of that at site $\mathrm{S} 1$, we observe larger velocity amplitudes in the east-west (fault perpendicular) direction for the slipand rate-weakening friction model but smaller amplitudes in the north-south (fault parallel) direction. Thus, for homogeneous initial shear strains we find little difference between the ground motions for the slip-weakening friction model (associated with cracklike behavior) and for the slip- and rate-weakening friction model (associated with pulselike behavior). As discussed below, this does not hold true for the case of surface rupture on a thrust fault or heterogeneous initial shear strains.

For the thrust fault we analyze the rupture dynamics and the resulting ground motions for the slip-weakening friction model and the slip- and rate-weakening friction model when we raise the fault so that the top sits at the ground surface. For scenario TH0/SW/U we use the same initial strain field and friction model parameters that we used for scenario TH8/SW/U where the top of the fault sits $8.0 \mathrm{~km}$ below the ground surface. For scenario TH0/SRW/U we increase the nominal maximum dynamic stress drop from 4.5 to $6.5 \mathrm{MPa}$ (at depths below $6.0 \mathrm{~km}$ ) in an attempt to create an earthquake with comparable slip to scenario TH8/SW/U. In order to maintain the same nominal minimum sliding shear stresses of $1.5 \mathrm{MPa}$, we increase the nominal initial shear tractions from 6.0 to $8.0 \mathrm{MPa}$ by scaling the tectonic strains given in equation (18) by a factor of 1.33 . We scale the distance from failure by the same amount to prevent substantial changes in the rupture speed. We use

$$
\begin{aligned}
& \mu_{\text {max }}= \begin{cases}0.222 & z>-1.0 \mathrm{~km} \\
-4.08 \times 10^{-3}\left(\frac{\mathrm{m}^{3} \mathrm{sec}^{2}}{\mathrm{~kg}}\right)^{1 / 2} \frac{\sqrt{\mu}}{z} & z<-1.0 \mathrm{~km}\end{cases} \\
& \mu_{\text {min }}= \begin{cases}0.0230 & z>-1.0 \mathrm{~km} \\
-4.22 \times 10^{-4}\left(\frac{\mathrm{m}^{3} \mathrm{sec}^{2}}{\mathrm{~kg}}\right)^{1 / 2} \frac{\sqrt{\mu}}{z} & z<-1.0 \mathrm{~km}\end{cases} \\
& \mu_{\text {post }}= \begin{cases}0.153 & z>-1.0 \mathrm{~km} \\
-2.04 \times 10^{-3}\left(\frac{\mathrm{m}^{3} \mathrm{sec}^{2}}{\mathrm{~kg}}\right)^{1 / 2} \frac{\sqrt{\mu}}{z} & z<-1.0 \mathrm{~km}\end{cases} \\
& D_{0}=0.446 \mathrm{~m} \\
& V_{0}=0.150 \mathrm{~m} / \mathrm{sec}
\end{aligned}
$$

for the parameters of the slip- and rate-weakening friction model in scenario TH0/SRW/U.

When slip occurs on the fault surface, the sliding stresses are relatively constant, and the fault acts like a free surface for normally incident shear waves. Consequently, after the seismic waves above the fault reflect off the ground surface and attempt to propagate back through the fault, most of the energy reflects off the fault surface and propagates back toward the ground surface; little energy is transmitted across the fault. The energy becomes trapped above the fault and creates severe ground motions near the surface rupture. Additionally, the normal stresses decrease ahead of the rupture, which reduces the distance to failure (Oglesby et al., 2000a,b). In scenario TH0/SW/U, beginning at $5.0 \mathrm{sec}$ and continuing until nearly $12.0 \mathrm{sec}$, we find much larger velocities above the fault than immediately below the fault. The extent of the dynamic interaction between the seismic waves and the slip on the fault depends on the instantaneous width of the rupture, that is, the area where slip is occurring. In scenario TH0/SRW/U the rate weakening in the friction model causes a narrow slip pulse that limits the area where the seismic waves may interact with the rupture. As a result, the rupture in scenario TH0/SRW/U yields an average slip of $1.1 \mathrm{~m}$, whereas the rupture in scenario $\mathrm{TH} 0 / \mathrm{SW} / \mathrm{U}$ yields a much larger average slip of $2.5 \mathrm{~m}$.

The maximum horizontal and vertical displacements along the north-south line across the top of the fault (Fig. 21) provide a good indication of the severity of the ground motions near the surface rupture. The entrapment of the seismic waves above the fault causes the severe motion to occur on the hanging-wall side of the fault. As documented by other researchers, such as Mikumo and Miyatake (1993) and Oglesby et al. (2000b) who use more traditional friction model parameters, the peak displacements display an asym- 
Site S1
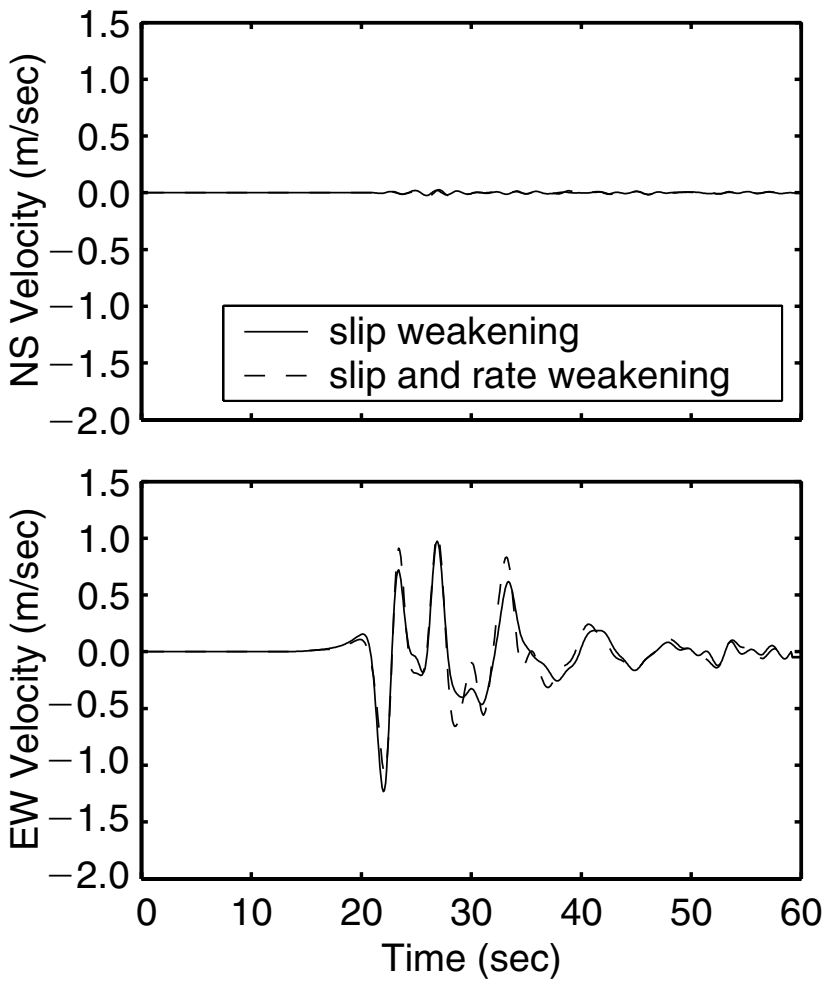

Site S2
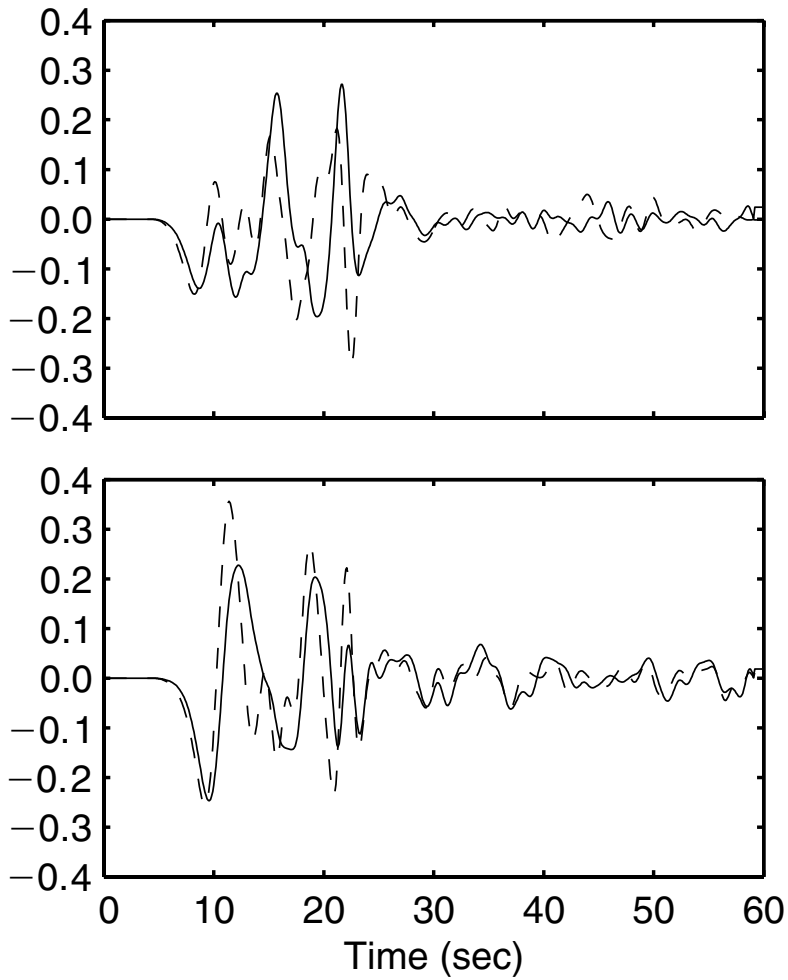

Figure 20. Comparison of horizontal velocity time histories at sites S1 and S2 in the strike-slip domain for the two friction models (scenarios SSO/SW/U and SSO/SRW/ $\mathrm{U})$. The slip-weakening friction model creates cracklike ruptures, and the slip- and rateweakening friction model creates pulselike ruptures. The time histories reflect the weak sensitivity of the ground motions to the type of friction model for uniform initial shear strains.
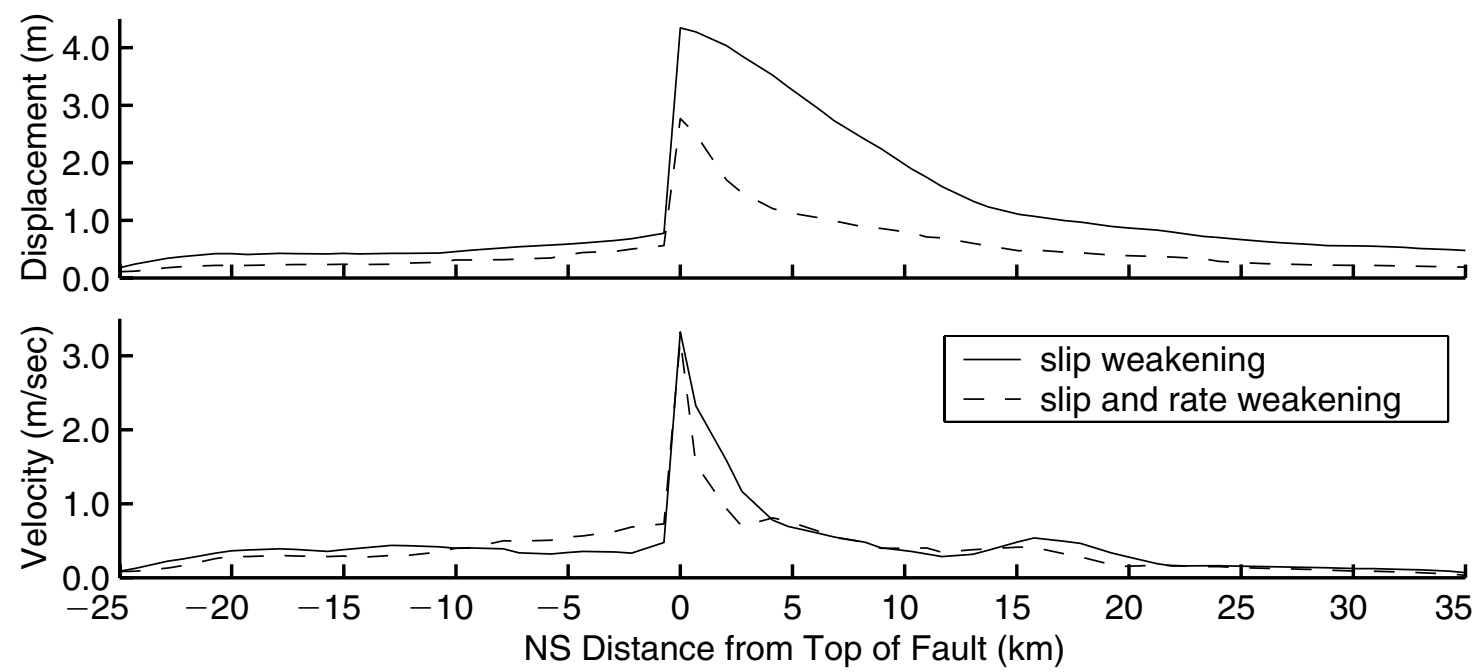

Figure 21. Peak horizontal particle displacements and velocities along a northsouth line running over the center of the thrust fault for the two friction models (scenarios $\mathrm{TH} 0 / \mathrm{SW} / \mathrm{U}$ and TH0/SRW/U). With initial shear strains that are independent of depth, the slip-weakening friction model associated with cracklike rupture yields very large shallow slips that produce large displacements on the hanging wall, whereas the slip- and rate-weakening friction model associated with pulselike rupture yields more moderate values. 
metry across the fault and exceed the static displacements. However, for both friction models the asymmetry in the static horizontal displacements across the fault at the ground surface exceeds the asymmetry in the peak horizontal displacements at the same locations by about $20 \%$. This is because the symmetric motion contributes to the peak displacements, and the peak values on opposite sides of the fault occur at different times. In scenario TH0/SW/U the peak maximum horizontal displacements exceed $2.0 \mathrm{~m}$ over an area of about $200 \mathrm{~km}^{2}$, with a peak value of $6.2 \mathrm{~m}$ at one location where the rupture hits the surface. Although the maximum vertical displacements do not reach the same levels as the maximum horizontal displacements, they do exceed $2.0 \mathrm{~m}$ along much of the surface rupture.

Consequently, although we still find larger velocities above the fault than below the fault in scenario TH0/SRW/ $\mathrm{U}$ compared to those in scenario $\mathrm{TH} 0 / \mathrm{SW} / \mathrm{U}$, the asymmetry across the fault occurs over a smaller area at any given time. Comparing the maximum horizontal displacements from scenario $\mathrm{TH} 0 / \mathrm{SW} / \mathrm{U}$ with those from scenario TH0/SRW/U in Figure 21, we see a substantial decrease in the values resulting from the smaller amount of dynamic interaction between the seismic waves and the rupture. While the largest motions continue to occur on the hanging wall, the largest peak horizontal displacement decreases from 6.2 to $4.0 \mathrm{~m}$. Similarly, the asymmetry (both static and peak displacements) across the fault decreases by approximately $20 \%$ from scenario $\mathrm{TH} 0 / \mathrm{SW} / \mathrm{U}$ to scenario TH0/SRW/U. Thus, the lack of any rate weakening in the slip-weakening friction model tends to accentuate the entrapment of the waves above the fault.

\section{Effect of Spatial Heterogeneity in the Parameters}

We gauge the sensitivity of the rupture behavior and the ground motions to heterogeneity in the initial shear tractions by introducing asperities into the tectonic shear strains. In scenario TH8/SW/HS the distribution of the initial shear tractions on the thrust fault buried $8 \mathrm{~km}$ below the surface contains 20 asperities with uniform random distributions of locations, radii between 3.0 and $8.0 \mathrm{~km}$, and heights between $+60 \%$ and $-60 \%$ of the nominal strain field (given by equation 18). Figure 22 shows the initial shear stresses on the fault surface. The slip-weakening friction model remains the same as the one in scenario TH8/SW/U, so we do not introduce any heterogeneity into the shear stresses at failure or the minimum sliding shear stresses.

We also create scenario TH8/SRW/HS that features a heterogeneous distribution of initial shear strains and a slipand rate-weakening friction model. This friction model exhibits very similar behavior to that of the slip- and rateweakening friction model used for the other scenarios. Aagaard (1999) gives a complete description of this friction model that is referred to as shear melting-refreezing. The friction model parameters closely match those of the slipand rate-weakening friction model in scenario TH0/SRW/U (equation 22). In order to create the appropriate nominal maximum dynamic stress drop of $6.5 \mathrm{MPa}$ that we need, with this friction model (which contains rate-weakening behavior), to generate slip comparable to that in scenario TH8/ $\mathrm{SW} / \mathrm{U}$, we superimpose the tectonic strain asperities from scenario TH8/SW/HS (which have an average value of zero) on the uniform tectonic strains from scenario TH0/SRW/U. Figure 22 displays the initial shear stresses applied to the fault surface.

We compare the distributions of final slip from scenarios TH8/SW/HS and TH8/SRW/HS with those from scenario TH8/SW/U (Fig. 17) to determine if the heterogeneous shear tractions introduce heterogeneity in the final slip. As shown in Figure 22, when we use the slip-weakening friction model, the heterogeneous tractions have little effect on the final slip distribution. Comparing the distributions of final slip for scenarios TH8/SW/U and TH8/SW/HS (Figs. 17 and 22), we find that the slip decreases in the upper-east corner of the fault (upper left-hand portion of the figure) in response to the smaller peak slip rates. In scenario TH8/SRW/HS (Fig. 22) we find a much stronger correlation between the distributions of final slip and the initial shear tractions. The larger slip rates with the slip- and rate-weakening friction model allow the rupture to generate the same slip over a shorter period of time that corresponds to a narrower rupture width. This localizes the slip, which enables the heterogeneity in shear tractions to create more heterogeneity in slip distribution. Furthermore, as shown in Figure 22, the large rupture width associated with the slip-weakening friction model smoothens the distribution of the initial shear tractions, whereas the narrow rupture width associated with the slip- and rate-weakening friction model roughens the initial shear tractions. Nielsen and Olsen (2000) observed this same behavior when they attempted to reproduce the rupture dynamics of the 1994 Northridge earthquake with a slip-weakening friction model and a slip- and rate-weakening friction model. This implies that the cracklike ruptures (slip-weakening friction) tend to create smooth distributions of slip and stress, whereas pulselike ruptures (slip- and rate-weakening friction) tend to create more heterogeneous distributions of slip and stress.

The peak horizontal velocities on the ground surface illustrate the effect of introducing heterogeneity into the initial shear tractions. The peak velocities at locations near regions with larger slip rates tend to increase. Figure 23 shows that the peak velocities increase above the southwest corner of the fault, compared with the distributions in Figure 18 for scenario $\mathrm{TH} 8 / \mathrm{SW} / \mathrm{U}$, because of the larger shear stresses near the top of the fault in the heterogeneous distributions of the initial shear tractions. On the other hand, the variation in the rupture speed tends to decrease the amplitude of the motion in other areas because it disrupts the reinforcement of the shear wave by the rupture. The slip- and rate-weakening friction model confines the increase in the peak displacements to smaller regions near the asperities that have a smaller distance from failure, so that, although the peak displacement increases from $0.68 \mathrm{~m}$ in scenario $\mathrm{TH} 8 / \mathrm{SW} / \mathrm{U}$ 

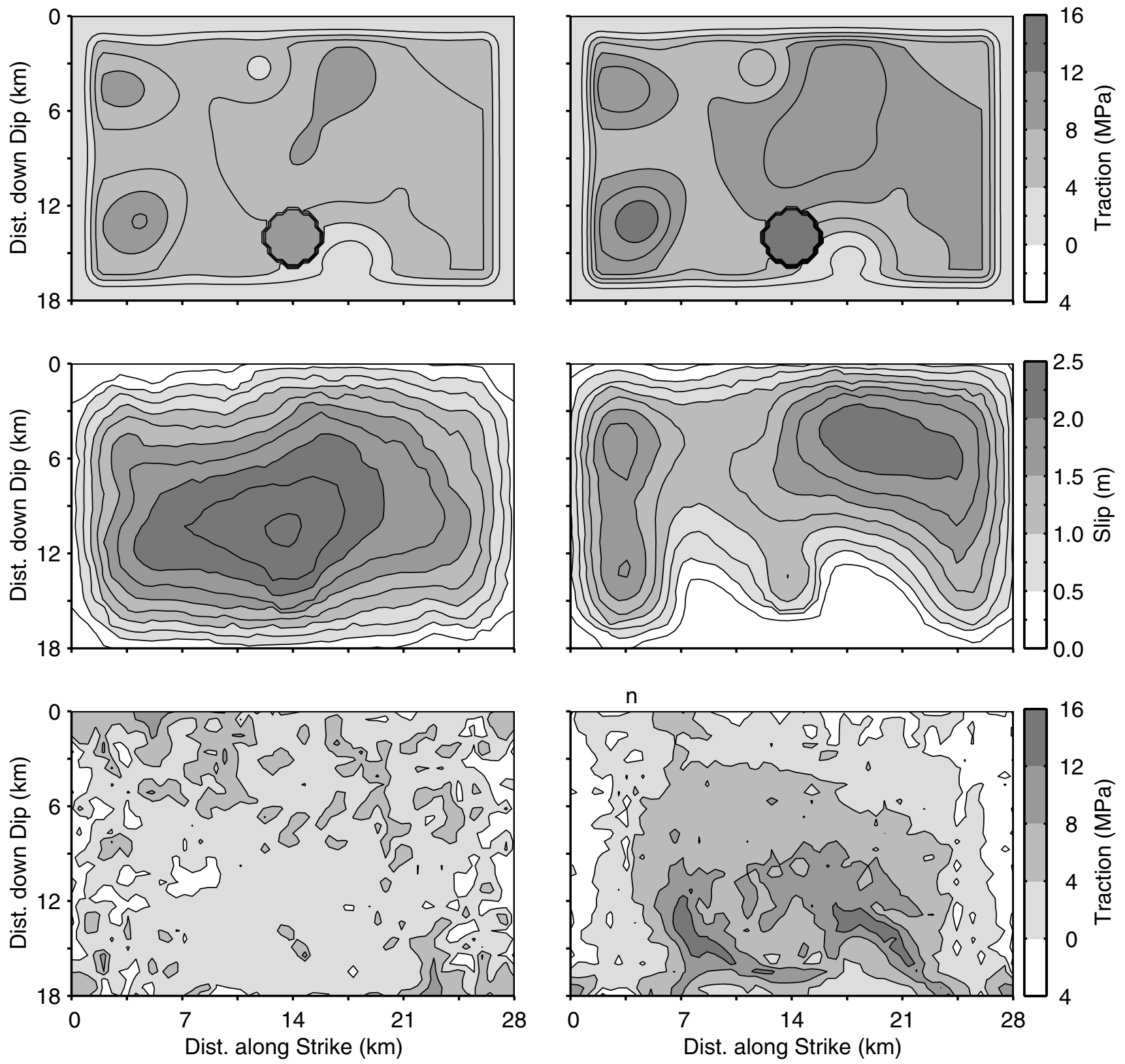

Figure 22. Initial shear tractions (top), final slip (middle), and final shear tractions (bottom) on the fault for scenarios TH8/SW/HS (left) and TH8/SRW/HS (right). The slip- and rate-weakening friction with a pulselike rupture produces heterogeneous distributions of slip and shear stress in contrast with the slip-weakening friction model with a cracklike rupture.

and $0.89 \mathrm{~m}$ in scenario $\mathrm{TH} / \mathrm{SW} / \mathrm{HS}$ to $1.2 \mathrm{~m}$ in scenario $\mathrm{TH} 8 / \mathrm{SRW} / \mathrm{HS}$, the area where the peak displacement exceeds $0.5 \mathrm{~m}$ actually drops from $810 \mathrm{~km}^{2}$ in scenario TH8/ SW/HS to $740 \mathrm{~km}^{2}$ in scenario TH8/MR/HS (albeit it remains considerably larger than the $480 \mathrm{~km}^{2}$ in scenario TH8/ $\mathrm{SW} / \mathrm{U})$.

Corresponding scenarios on the strike-slip fault produce similar behavior. The initial shear tractions generate greater heterogeneity in the slip distribution when the friction model includes rate-weakening behavior. Additionally, the effects of encountering variations in the initial shear tractions are more evident because of the longer length of the strike-slip fault. The peak velocities increase as the rupture encounters higher initial shear stresses, and the peak slip rates and the rupture speed both increase. The peak velocities then rapidly decrease as the peak slip rates drop, and the rupture slows down as the initial shear tractions return to more moderate levels.

We can also create variations in the distributions of final slip and peak slip rate by introducing heterogeneity into the 

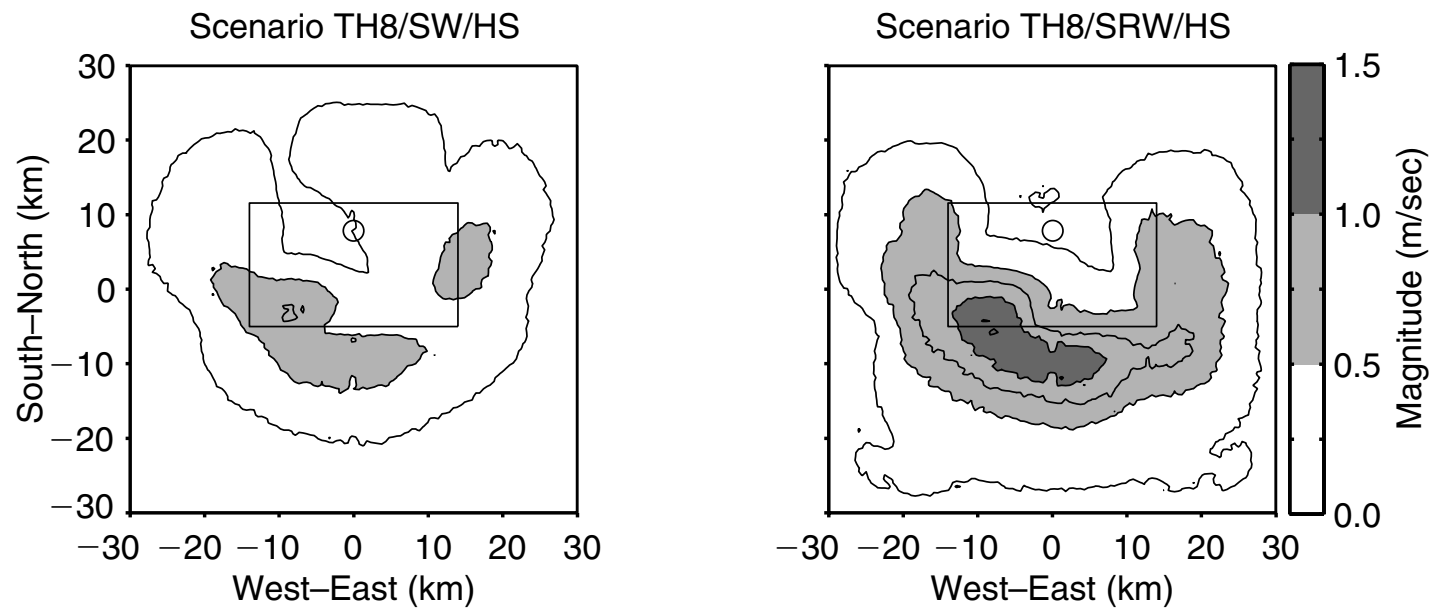

Figure 23. Peak horizontal particle velocities on the ground surface for scenarios TH8/SW/HS and TH8/SRW/HS. The line indicates the projection of the fault plane on to the ground surface, and the hollow circle identifies the epicenter. The reduced distance to failure near the top of the fault in the heterogeneous initial shear tractions for both scenarios produces larger ground motions above the southwest portion of the fault when compared with scenario TH8/SW/U (Fig. 18).

parameters of the slip-weakening friction model. We follow the same procedure that we used for creating heterogeneity in the initial shear strains and place the 30 asperities on the strike-slip fault using uniform random distributions. The radii vary from 3.0 to $8.0 \mathrm{~km}$, and the asperity heights correspond to variations in the coefficients in the friction model. We independently vary the coefficients in the expressions for $\mu_{\max }$ and $\mu_{\min }$ by up to $40 \%$ above and below their nominal values. We do not introduce heterogeneity in the characteristic slip distance; therefore, the fluctuations in the maximum and minimum values of the coefficient of friction also cause heterogeneity in the fracture energy. Following our scenario nomenclature, we designate this scenario SSO/ $\mathrm{SW} / \mathrm{HF}$.

As in the case of the heterogeneous initial shear strains, the heterogeneity in the friction model parameters has a greater impact on the peak slip rate than the final slip. The regions with the larger peak slip rates correlate with the regions of a reduced distance from failure, and vice versa. In the central portion of the strike-slip fault we observe a relatively uniform increase in slip corresponding to the reduced distance from failure. This leads to the larger peak velocities near the central portion of the fault as shown by comparing Figure 24 with Figure 14. We found similar trends in scenario TH8/SW/HS, which used heterogeneous initial shear stresses and a slip-weakening friction model. Whereas the average slip of $2.0 \mathrm{~m}$ in scenario SSO/SW/HF nearly matches the average slip of $1.9 \mathrm{~m}$ in scenario $\mathrm{SS} 0 / \mathrm{SW} / \mathrm{U}$, the heterogeneity in the friction model parameters decreases the maximum peak horizontal displacement on the ground surface by $20 \%$ and the maximum peak horizontal velocity by $11 \%$.
Transparent Versus Nontransparent Fault Conditions

When we raise the top of the thrust fault to the ground surface, the sliding stress boundary conditions on the fault during the dynamic rupture trap the seismic waves above the fault. In traditional Haskell-type source models (Haskell, 1969), such as prescribed ruptures, the shear waves propagate across the fault zone as if it did not exist ("transparent fault conditions"). However, in a real earthquake and in the dynamic ruptures discussed earlier, when a shear wave attempts to propagate across the fault at a place that is sliding, the seismic wave interacts with the fault slip. In the extreme case that the fault is sliding at a constant level of friction, the fault surface prevents the transmission of shear waves propagating normal to the fault. In order to include the effects of the interaction in a prescribed rupture, the rupture behavior must be specified to mimic the slip history produced by this interaction. The complex nature of the interaction makes this difficult to do.

We compare the dynamic rupture scenarios $\mathrm{TH} 0 / \mathrm{SW} / \mathrm{U}$ and TH0/SRW/U with the prescribed rupture scenario THO/ $\mathrm{P} / \mathrm{U}$ to see how the inclusion of the dynamic interaction changes the ground motions. For the source parameters in the prescribed rupture scenario $\mathrm{TH} / \mathrm{P} / \mathrm{U}$, we use the distributions of final slip and peak slip rate from scenario $\mathrm{TH} 0 /$ SRW/U (pulselike rupture with slip- and rate-weakening friction) along with a uniform rupture speed of $80 \%$ of the local shear-wave speed, so that the only major difference between scenarios TH0/SRW/U and TH0/P/U is the transparent fault conditions in scenario TH0/P/U. For scenario THO/SW/U (cracklike rupture and slip-weakening friction) the average peak slip rate of $1.1 \mathrm{~m} / \mathrm{sec}$ matches that in scenario TH0/SRW/U, but the average slip of $2.5 \mathrm{~m}$ is much larger than that for scenarios TH0/SRW/U and TH0/P/U. 
As mentioned in our discussion of surface rupture on the thrust fault with the different friction models, the degree to which the dynamic interaction occurs depends on the downdip width of the rupture at any point in time. The slipweakening friction model, which tends to create wide ruptures, accentuates this effect as previously illustrated in Figure 21 and reproduced in Figure 25. Even though the distributions of final slip and peak slip rate in the prescribed rupture are identical to those in the scenario with slip- and rate-weakening friction, the transparent fault conditions in the prescribed rupture prevent the trapping of energy in the hanging wall and do not result in any significant increase in the peak horizontal velocities approaching the top of the fault. Whereas the peak horizontal displacements do increase slightly on the hanging wall near the top of the fault, the motions for the pulselike rupture (slip- and rate-weakening friction) diverge from those of the prescribed rupture to much larger values within $3 \mathrm{~km}$ of the top of the fault. Fur- thermore, comparing scenarios $\mathrm{TH} / \mathrm{P} / \mathrm{U}$ and $\mathrm{TH} 0 / \mathrm{SRW} / \mathrm{U}$ shows that the transparent fault conditions in scenario $\mathrm{TH} 0 /$ $\mathrm{P} / \mathrm{U}$ reduce the asymmetry in the peak horizontal displacements across the fault at the ground surface by about $15 \%$. Thus, we find that the dynamic interaction between the seismic waves and the rupture (nontransparent fault conditions) tends to increase the ground motions near the surface rupture on the hanging wall.

Brune (1996) suggested that this trapping of the energy in the hanging wall of the fault may lead to systematic underestimation of the shallow slip on thrust faults in source inversions (which use transparent fault conditions) because of the lack of energy radiated to teleseismic distances. We consider displacement time histories in the center of the domain at the surface $(5 \mathrm{~km}$ north of the top of the fault) and at a depth of $20 \mathrm{~km}$ in order to determine if the motions below the fault at depth, which propagate to teleseismic distances, display features that would lead to an underestima-

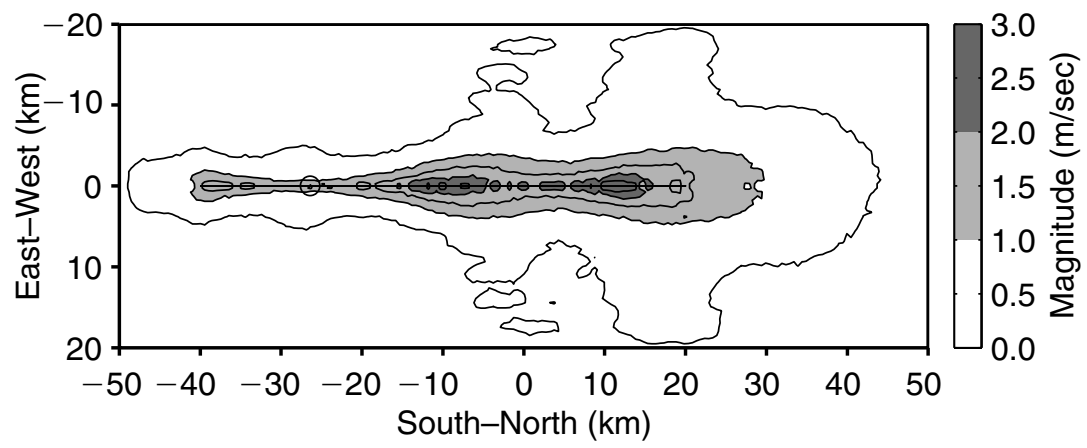

Figure 24. Peak horizontal particle velocities on the ground surface for scenario SSO/ $\mathrm{SW} / \mathrm{HF}$. The line indicates the projection of the fault plane on to the ground surface, and the hollow circle identifies the epicenter. The peak velocities increase in regions where the fault is closer to failure and decrease in regions where the fault is farther from failure compared with the case of only vertical variation of the friction parameters (Fig. 14).
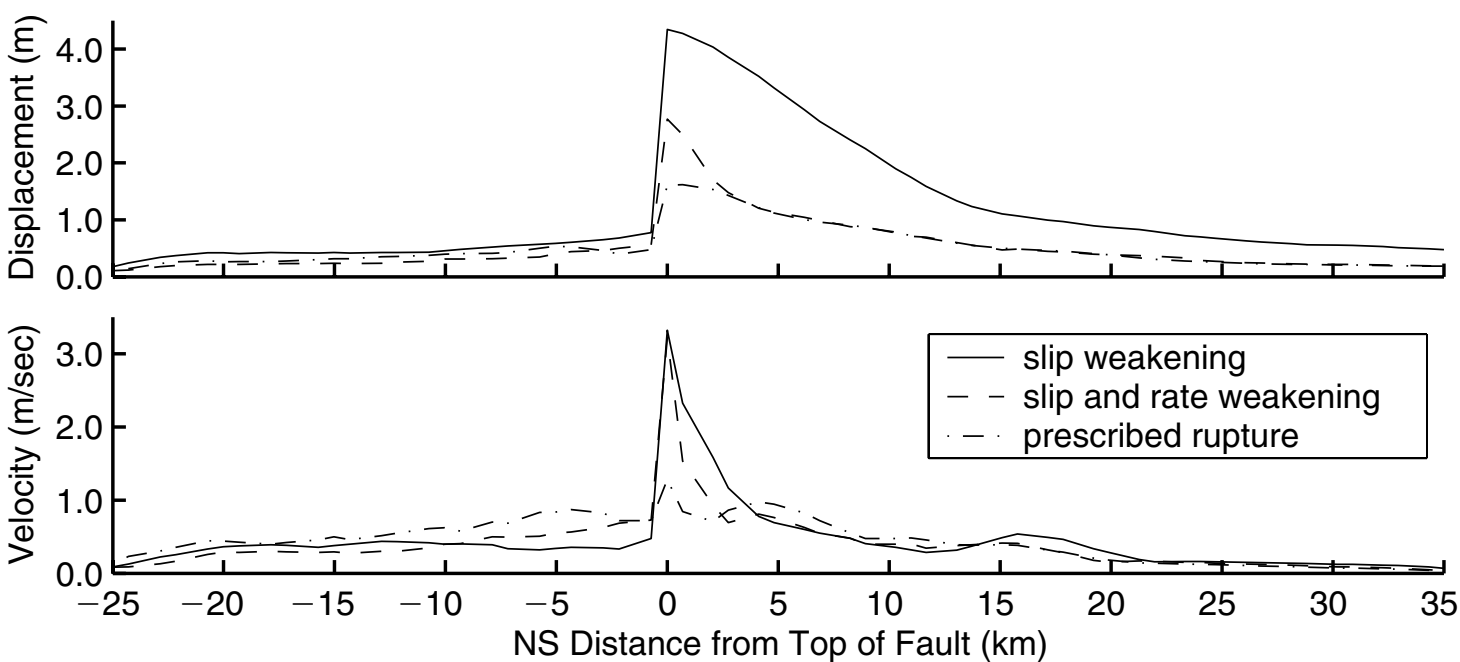

Figure 25. Peak horizontal particle displacements and velocities along a northsouth line running over the center of the thrust fault for dynamic rupture scenarios $\mathrm{TH} / \mathrm{SW} / \mathrm{U}$ and TH0/SRW/U (nontransparent fault conditions) and prescribed rupture scenario TH0/P/U (transparent fault conditions). For the dynamic ruptures the peak velocities increase on the hanging wall approaching the top of the fault, whereas those from the prescribed rupture display no significant increase. Similarly, the peak displacements for the dynamic ruptures increase at a faster rate on the hanging wall near the top of the fault compared with the prescribed rupture. 
tion of slip. We normalize the displacement amplitudes in Figure 26 relative to the average slip in order to allow comparison between the cracklike rupture with the slip-weakening friction model and the pulselike rupture with the slipand rate-weakening friction model. Based on Figure 25, the north-south displacement time histories on the ground surface in Figure 26 for the prescribed rupture TH0/P/U exhibit the expected close agreement with those for scenario $\mathrm{TH} 0 /$ SRW/U because of the matching of the distributions of final slip and peak slip rate. Additionally, the larger amount of dynamic interaction in scenario THO/SW/U leads to slightly larger motions relative to the average slip at the ground surface. In all three cases the normalized amplitudes lie near unity so that the displacement amplitudes roughly match the average slip.

Below the center of the fault, the largest motions on the north-south component correspond to the $s S$ phase. In contrast to the motions at the ground surface, at a depth of 20 $\mathrm{km}$ the peak north-south displacement for the dynamic rupture with slip- and rate-weakening friction is $17 \%$ smaller than that for the prescribed rupture scenario. The waveforms for all three scenarios have similar shapes, but the waveform for the dynamic rupture with slip-weakening friction has a slow onset of motion so that the peak amplitude is delayed in time. On the basis of the smaller amplitudes with the similarly shaped waveforms, one would incorrectly infer slightly smaller values of average slip for the dynamic ruptures that have nontransparent fault conditions. The vertical component corresponding to the $s P$ phase exhibits similar behavior. This means that source inversions of teleseismic waveforms with transparent fault conditions may tend to underestimate the amount of shallow slip by a small amount.

\section{Discussion}

We generate reasonable ruptures on strike-slip and thrust faults in a homogeneous half-space and a layered halfspace by making the parameters in the friction model a function of either the ratio of the square root of the shear modulus to the depth or the ratio of the shear-wave speed to the depth. Apart from the tapering in slip along the buried edges of the fault, the distributions of slip exhibit no clear trends with depth, so they are compatible to first order with those observed in source inversions. The average peak slip rates also fall into the appropriate range of about $1-2 \mathrm{~m} / \mathrm{sec}$. Although we do observe variations in the rupture speed as the rupture encounters changes in the distance from failure (through heterogeneity in either the initial shear tractions or the fracture energies), the ruptures generally propagate below the shearwave speed in accordance with source inversions.

In order to generate slip distributions with no clear trends with depth, the absolute change in the coefficient of friction must decrease with depth because the effective normal stresses increase with depth. The variation of the coefficient of friction is also constrained by assuming initial shear tractions derived from a uniform strain field. Under these conditions our choice of making the effective coefficient of friction a function of the depth and the shear modulus creates distributions of fracture energy and dynamic stress drop that produce slip distributions with no clear depth dependence and limit temperature changes on the fault surface to ranges that do not imply significant melting. This results in coefficients of friction that range from 0.2 at failure to 0.02 during sliding at $1-\mathrm{km}$ depth and from 0.03 at failure to 0.005 during sliding at $15-\mathrm{km}$ depth. Recall that the co-
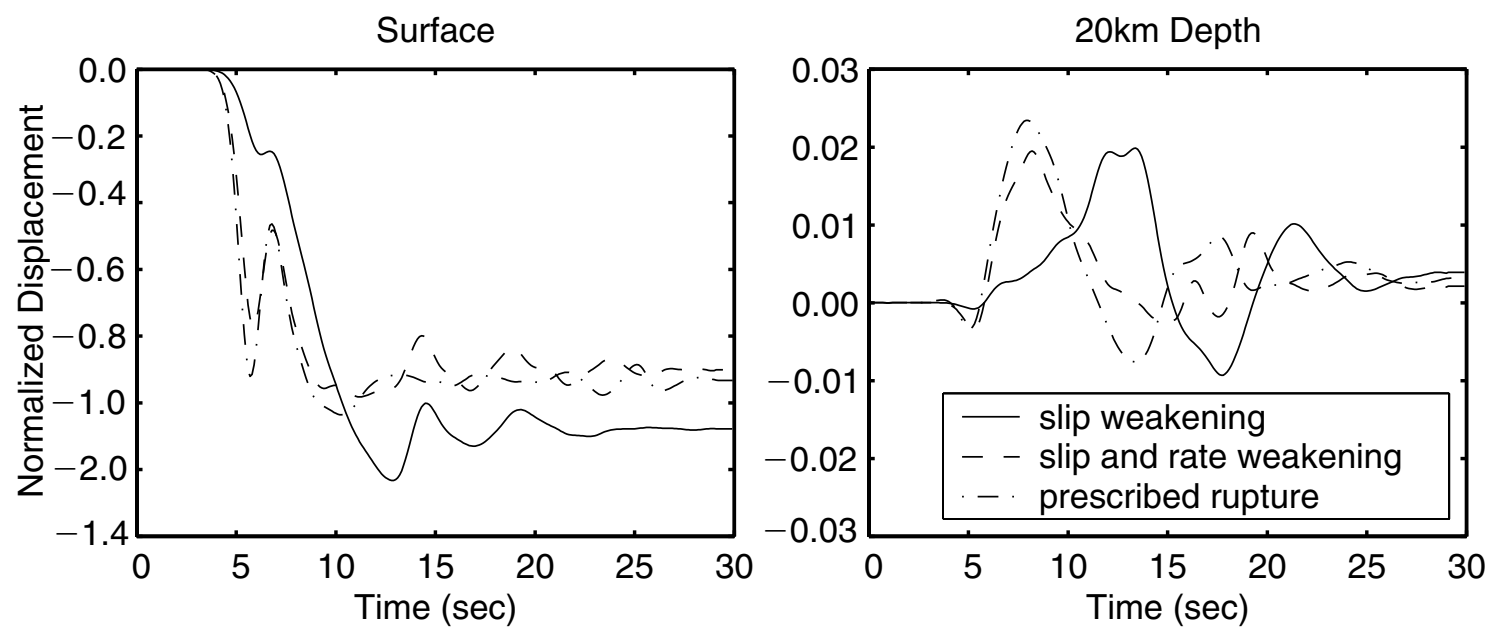

Figure 26. Normalized north-south displacement time histories in the center of the domain at the ground surface and at a depth of $20 \mathrm{~km}$ for dynamic rupture scenarios $\mathrm{TH} 0 / \mathrm{SW} / \mathrm{U}$ and $\mathrm{TH} 0 / \mathrm{SRW} / \mathrm{U}$ (nontransparent fault conditions) and prescribed rupture scenario TH0/P/U (transparent fault conditions). The amplitudes are normalized with respect to the average slip. At depth, the displacement amplitudes differ only slightly for the dynamic ruptures relative to the average slip from those for the prescribed rupture (Haskell-type source). 
efficient of friction at failure strongly depends on the discretization size; the fracture energy of around $1.5 \mathrm{MJ} / \mathrm{m}^{2}$ provides a more physically meaningful parameter for the initiation of sliding (failure). This fracture energy exceeds the values from laboratory measurements for extension of a crack in polycrystalline materials by four orders of magnitude (Lawn, 1993), making the interpretation of this large fracture energy problematic. We can only speculate that a significant amount of anelastic deformation occurs at the leading edge of large earthquake ruptures.

Whereas the friction models and the variation of the parameters with the material properties and the depth are not based on a particular mechanism for the sliding friction, a number of mechanisms have been proposed for low levels of dynamic friction during earthquakes (Brune et al., 1993; Melosh, 1996; Harris and Day, 1997; Sleep, 1997; Tworzydlo and Hamzeh, 1997; Ben-Zion and Andrews, 1998; Anooshehpoor and Brune, 1999; Brodsky and Kanamori, 2001). A combination of such mechanisms may produce a complex variation in the effective coefficient of friction over the depth of the fault during sliding, which resembles our ad hoc variation. Moreover, we could formulate the friction stress for the slip-weakening friction model, which is independent of the normal stress, by using a low-sliding friction stress instead of a minimum coefficient of friction, and a drop in the friction stress over a slip-weakening distance as a proxy for the fracture energy; this would lead to similar results with, arguably, a more physically meaningful friction model.

Such a friction model would eliminate the need to clip the coefficient of friction to reasonable values to prevent it from approaching infinity at shallow depths. Additionally, preliminary analyses indicate that it tends to eliminate two features present in some of our simulations that do not seem to be observed in real earthquakes: the large peak slip rates and the final slips in a narrow region along the free surface. In the strike-slip simulations these features may be attributed to the decrease in fracture energies in the softer material near the surface that arise from the formulation of the friction force with a coefficient of friction coupled with low effective normal stresses. However, on the thrust fault the dynamic interaction between the seismic waves and the rupture also contributes to the large peak slip rates and final slips near the surface. As discussed above, the choice of friction model affects the width of the rupture and strongly influences the extent of the dynamic interaction, and hence the slip rate and final slip at shallow depths.

\section{Type of Friction and Heterogeneous Fault Tractions}

On both faults the replacement of the slip-weakening model (associated with cracklike ruptures) with a slip- and rate-weakening friction model (associated with pulselike ruptures) in the presence of a heterogeneous initial shear strain field leads to a substantial increase in the heterogeneity in the distribution of the final slip. These local changes in slip and slip rate alter the shapes and amplitudes of the ground-motion time histories. Thus, we find that the ground motions exhibit a strong sensitivity to the degree of heterogeneity, particularly when the friction model contains rateweakening behavior (pulselike ruptures). These observations, along with similar ones by Nielsen and Olsen (2000), suggest that rate weakening likely plays an important role in generating heterogeneous slip distributions and support the theory of Madariaga and Cochard (1996) that any friction model that produces a large dynamic stress drop compared with the average final stress drop will produce heterogeneity in the final shear stress; ultimately, this leads to slip heterogeneity. In other words, the conclusion that rate weakening likely influences the heterogeneity in the distribution of slip does not explicitly depend on our choice of the values for the coefficient of friction and normal stress; it only depends on the dynamic stress drop.

On the basis of kinematic inversions of several recent earthquakes, Beroza and Mikumo (1996), Bouchon (1997), and Day et al. (1998) found that small-scale spatial variations (many of the asperities had radii less than $1 \mathrm{~km}$ ) in the dynamic stress drop alone may generate the heterogeneity in the final slip. Our use of larger asperities $(3.0-8.0 \mathrm{~km}$ in radius) in the initial shear stresses may not provide sufficient heterogeneity in the dynamic stress drop to arrest the rupture at a local level when we use the slip-weakening friction model. As a result, the distributions of final slip closely resemble the final slip from a uniform stress drop earthquake. Thus, we cannot dismiss the possibility that the slip-weakening friction model (which creates cracklike ruptures) with greater heterogeneity in the initial shear tractions will produce the same level of heterogeneity in the distribution of final slip that is generated by a friction model that contains rate-weakening behavior (which creates pulselike ruptures). However, because the ruptures with the slip-weakening friction model tend to smoothen the distribution of the initial shear tractions, whereas the ruptures with slip- and rateweakening friction tend to maintain heterogeneity in the shear tractions, the slip- and rate-weakening friction model would appear to be able to maintain heterogeneity in the shear tractions and distributions of slip over successive events on a fault surface, whereas the slip-weakening friction model would lead to migration toward homogeneous shear tractions and distributions of slip.

\section{Type of Friction and Nontransparent Fault Conditions}

When we raise the top of the thrust fault to the ground surface, the dynamic interaction between the relatively constant sliding stresses on the fault and the seismic waves above the fault causes large displacements and velocities on the ground surface of the hanging wall. Shi et al. (1998) observed similar behavior in domains with homogeneous material properties during simulations of dynamic rupture on a low-angle thrust fault using a two-dimensional solid lattice model with confining pressures that increase with depth, as did Oglesby et al. (2000b) with a three-dimensional finite-element model and uniform normal stresses. The slip- 
weakening friction model accentuates this effect because it tends to create wide ruptures. Using the slip- and rate-weakening friction model, we observe significantly less dynamic interaction, and the asymmetry in the peak horizontal displacements across the fault at the ground surface decreases. Nevertheless, in both cases the asymmetry in the peak horizontal displacements exceeds that found in a prescribed rupture with transparent fault conditions that has the same distributions of final slip and peak slip rate as the scenario with slip- and rate-weakening friction. However, as discussed above, the use of a coefficient of friction with lithostatic normal stresses may also accentuate these near-surface effects.

The displacement time histories below the center of the fault (which we associate with the waves radiated to teleseismic distances) for both the slip-weakening and slip- and rate-weakening friction models display slightly smaller amplitudes relative to the average slip compared with those of a prescribed rupture with a traditional Haskell-type source. This suggests that source inversions that do not account for the dynamic interaction would infer slightly less slip at shallow depths for thrust faults. Consequently, these simulations provide some support for the underestimation of shallow slip in source inversions that rely on teleseismic data as suggested by Brune (1996). However, a more complete understanding of the effect of transparent fault conditions on teleseismic waves will require propagating the seismic waves to large distances. This can be accomplished by calculating the teleseismic waveforms resulting from double-couples (with transparent fault conditions), where the spatial and temporal distribution of slip is derived from dynamic rupture models (with nontransparent fault conditions).

\section{Rupture Speed Relative to Slip Direction}

In the dynamic rupture simulations the speed of the rupture depends on the direction of propagation relative to the slip direction. As documented by other researchers, such as Andrews (1976b), Day (1982b), and Madariaga et al. (1998), the rupture propagates at just below the Rayleighwave speed in the direction of slip, but at a slower speed in the direction perpendicular to slip. The absolute and relative speeds in the two directions depend on the fracture energy, but we consistently observe a $20 \%$ reduction in the speed of the rupture in the direction perpendicular to the slip compared with the direction parallel to the slip. In prescribed rupture simulations we generally assume a uniform rupture speed relative to the shear-wave speed. This difference is minimal on long, narrow faults where the direction of slip coincides with the longer dimension (strike-slip faults) because the rupture propagates predominantly in one direction along the fault at just below the Rayleigh-wave speed. However, when the slip direction is nearly perpendicular to the longer dimension, as is generally the case on long thrust and normal faults, the rupture propagates at close to the Rayleigh-wave speed along the shorter dimension but at a slower speed along the longer dimension. This tends to create more bilateral-type ruptures, which decreases the amplitudes of the ground motions (Aagaard, 1999).

\section{Average Stress Drop and Average Slip}

Using the strike-slip simulations and the thrust fault simulations with a buried fault, we consider how well our earthquakes in the layered half-space fit the proportionality between average stress drop and average slip given by

$$
\Delta \bar{\sigma}=C \mu \frac{\bar{D}}{w},
$$

which applies to homogeneous half-spaces. Using the data from the dynamic rupture scenarios discussed earlier, supplemented by the five additional strike-slip scenarios and six additional thrust fault scenarios discussed in Aagaard (1999), Figure 27 shows that the proportionality continues to provide a good description of the relationship. The lines indicate the average proportionality between the average stress drop and the average slip for the simulations on the strike-slip fault and the thrust fault (each symbol corresponds to one scenario). If we use the shear modulus from a depth of $6.0 \mathrm{~km}$, the lines correspond to $C=0.45$ and $C=1.5$ in equation (23) for the strike-slip fault and the thrust fault, respectively. The value of $C=0.45$ for the strike-slip fault falls below the value of $C=0.7$ from equation (14). We attribute the difference to the variation of the maximum dynamic stress drop with depth and the tendency for the larger slips to occur near the ground surface where the shear modulus and, consequently, the stress drop are smaller. For buried thrust faults no relationships have been found relating the average stress drop to the average slip as a function of the fault geometry and the depth of the top of the fault. As noted by Parsons et al. (1988), the proportionality constant should be less for a thrust fault than for the

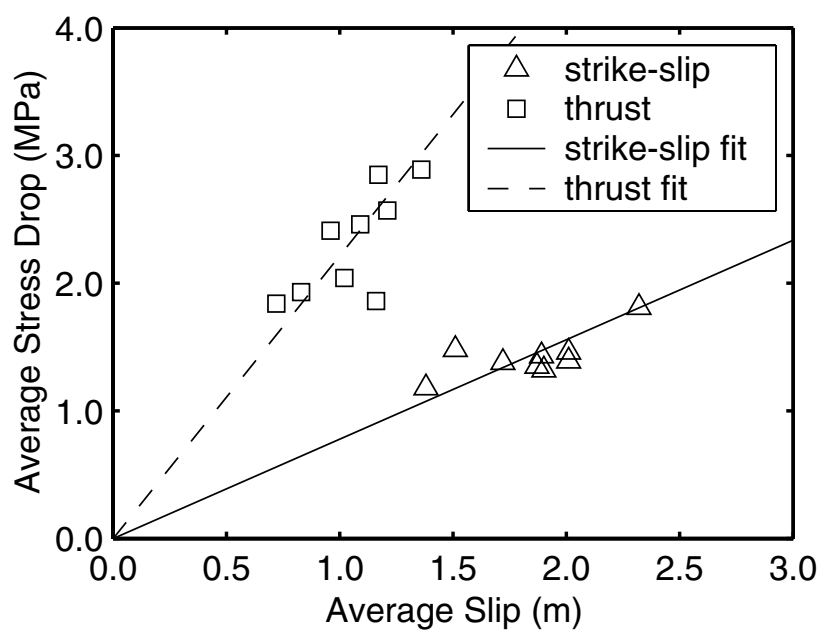

Figure 27. Average stress drop as a function of average slip for the scenarios with the strike-slip fault (triangles) and the thrust fault (squares). The solid and dashed lines indicate the linear fit for each fault. 
strike-slip fault of the same size, because of the relatively smaller stiffness above a thrust fault compared with the stiffness below. Equation (14) yields a value of $C=1.6$ for a deeply buried strike-slip fault with the same dimensions as our thrust fault. Consequently, our value of $C=1.5$ falls slightly below that of the strike-slip fault and is consistent with the numerical results of Parsons et al.

\section{Energy Balance and Seismic Efficiency}

We can compute the energy balance for each dynamic rupture event as described earlier in the Methodology section. Recall that restricting our domains to only a small fraction of the Earth limits the terms in the energy balance to the change in potential energy, the radiated energy, and the change in thermal energy (which includes the change in heat energy and the fracture energy). We model only the longperiod motion and do not include the energy radiated at short periods. Because we can determine the general behavior of the rupture from ground motions using source inversions, the radiated energy and fracture energy are well constrained by data from real earthquakes. If we maintain the same dynamic stress drop and distance from failure (fracture energy), but reduce the absolute level of the shear stresses, the rupture behavior does not significantly change. This leads to smaller changes in the heat energy and the potential energies, with no change in the radiated energy and the fracture energy. Thus, the seismic efficiency becomes larger as the dynamic friction (heat energy) decreases. This means that using dynamic friction stresses consistent with the lack of significant melting on the fault places a lower bound on the seismic efficiency.

In Figure 28 we display a typical energy balance for a strike-slip simulation and a thrust fault simulation. For these two scenarios the rupture speeds in the mode-II direction of $91 \%$ and $88 \%$ of the local shear-wave speed fall close to the average rupture speed observed for real earthquakes (Heaton, 1990). On both faults the total change in thermal energy (sum of the change in thermal energy and the fracture energy) exceeds the (long-period) radiated energy by a factor of approximately 1.8; this corresponds to a seismic efficiency of $36 \%$. For a mode-III crack propagating at a speed of $V_{\mathrm{r}}$,

$$
\eta=\left(1-\sqrt{\frac{1-\frac{v_{\mathrm{r}}}{\beta}}{1+\frac{v_{\mathrm{r}}}{\beta}}}\right)\left(\frac{1}{1+\frac{Q_{\mathrm{temp}}}{\Delta W}}\right)
$$

gives the seismic efficiency $(\eta)$ as a function of the shearwave speed $(\beta)$, the heat energy $\left(Q_{\mathrm{temp}}\right)$, and the change in potential energy $(\Delta W)$ (Kanamori et al., 1998; Kanamori and Heaton, 2000). Using the rupture speed in the mode-III direction (which is approximately $80 \%$ of the rupture speed in the mode-II direction), equation (24) predicts seismic efficiencies that are about $20 \%$ larger than those of the dynamic

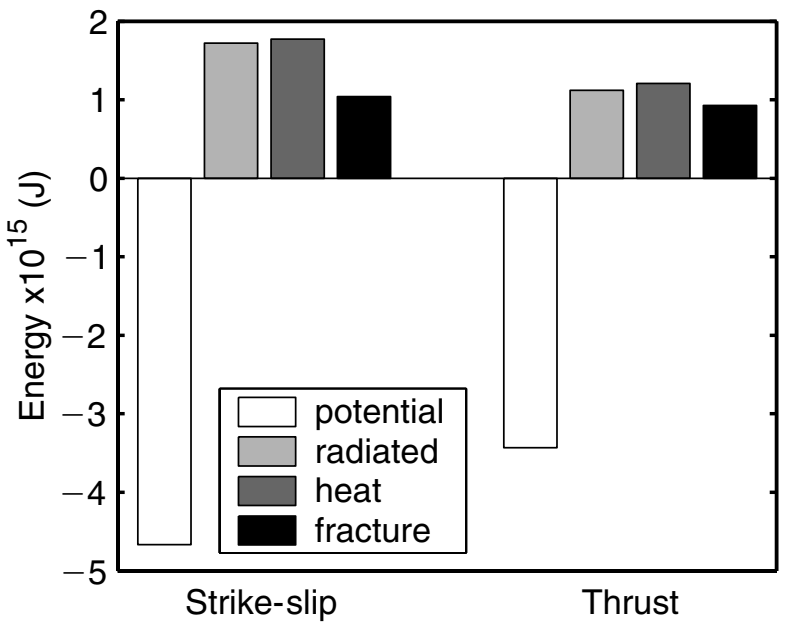

Figure 28. Energy balances for a typical strikeslip scenario and a fault scenario. The radiated energy accounts for approximately one-third of the decrease in the potential energy. This represents a lower bound because constraints on the rupture speed and temperature changes place only upper bounds on the fracture energy and the change in heat energy.

ruptures. The discrepancy between the values from this equation and those in the dynamic rupture simulations likely comes from the fact that the simulations contain only the long-period radiated energy. Including the energy radiated at shorted periods in the simulations would increase the seismic efficiency and bring it more in line with the relationship given by equation (24).

With the radiated energies and moment magnitudes of each event, we see how our events fit the Gutenberg-Richter relationship between radiated energy and magnitude. The Gutenberg-Richter energy-magnitude relation gives the radiated energy in ergs in terms of the surface wave magnitude, $M_{\mathrm{S}}$. Using the expressions for the surface wave magnitude and moment magnitude as a function of the seismic moment, we manipulate the energy-magnitude relation to give the radiated energy in joules in terms of the moment magnitude:

$$
\log _{10} E(J)=11.75+1.5 \mathbf{M}-7 .
$$

Again, supplementing the scenarios discussed here with six additional strike-slip scenarios and 11 additional thrust scenarios from Aagaard (1999) (including both buried thrust fault scenarios and those with surface rupture), Figure 29 shows that the thrust events lie below the Gutenberg-Richter relationship, whereas the strike-slip events all lie above. Thrust fault scenario TH0/SW/U with surface rupture and the slip-weakening friction model (the rectangle on the bottom right) does not fit the relationship because the large area where the dynamic interaction occurs between the rupture and the seismic waves generates much larger slips relative to other earthquakes with the same radiated energy. The dif- 

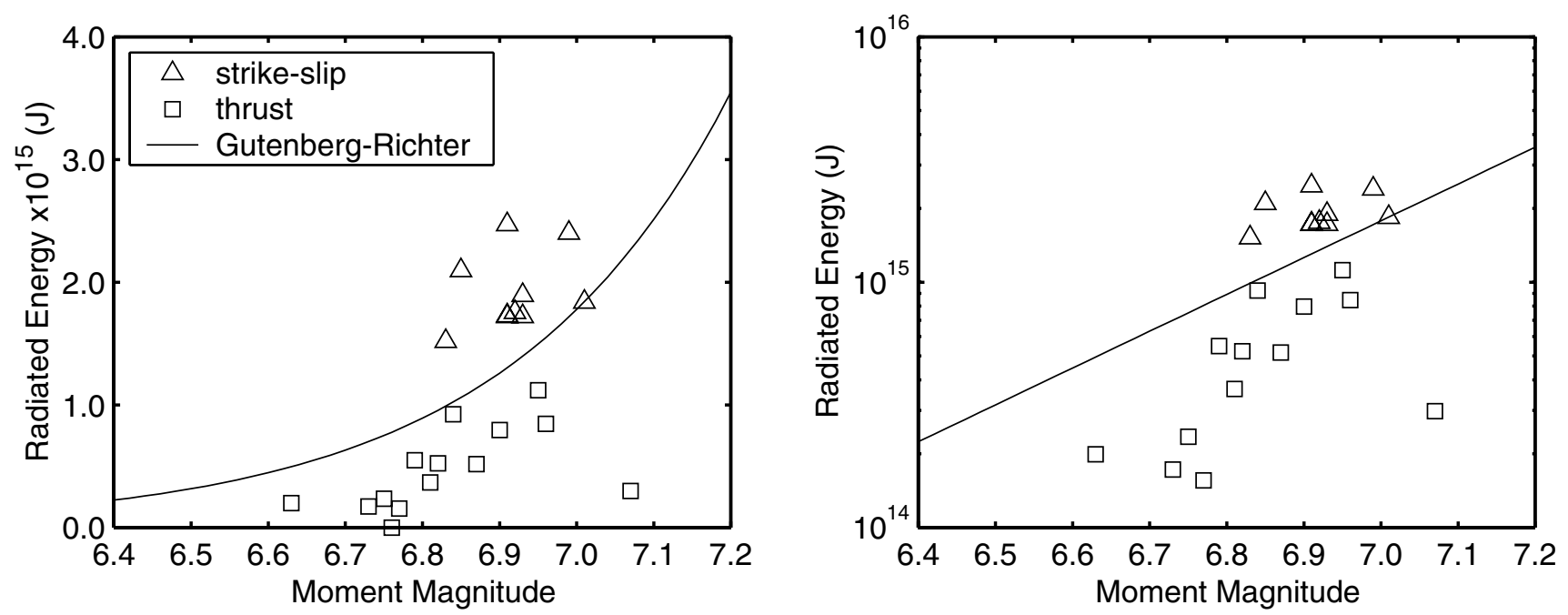

Figure 29. Radiated energy as a function of moment magnitude for the strike-slip (triangles) and thrust (squares) earthquake simulations. Variations in the average peak slip rate create the scatter of the simulations about the Gutenberg-Richter relationship. The rectangle at the bottom right corresponds to thrust fault scenario TH0/SW/U (surface rupture and the slip-weakening friction model) and does not appear to fit the relationship.

ferent average peak slip rates and rupture speeds create the scatter in the distribution of the radiated energies. We find relatively smaller slip rates for buried ruptures compared with surface ruptures, and relatively smaller slip rates for ruptures that begin near an edge of the fault. Both these factors contribute to the thrust fault scenarios consistently falling below the strike-slip fault scenarios. Thus, most of the scatter about the Gutenberg-Richter relationship results from variations in the average peak slip rates and rupture speeds.

\section{Conclusions}

Simulations on a strike-slip fault and a thrust fault demonstrate that in order to generate reasonable slip distributions with realistic effective normal stresses (the normal stresses increase with depth because of gravity), the change in stress on the fault must follow the variation of the shear modulus, and the fracture energy must be relatively uniform with the depth. We found that assuming that the coefficient of friction varies inversely with depth and proportionally with either the square root of the shear modulus or the shear-wave speed creates the appropriate variation of the stress drop with depth and keeps the fracture energy relatively independent of depth over much of the fault. Additionally, this formulation yields temperature changes on the fault that are consistent with the lack of significant melting observed in exposed fault zones. As a result, the ruptures have reasonable behavior in terms of general trends in the distribution of slip, the peak slip rate, and the rupture speed. In the direction of slip, the ruptures generally propagate at speeds between $50 \%$ and $90 \%$ of the shear-wave speed, whereas in the direction perpendicular to slip the ruptures propagate approximately $20 \%$ slower.

On both faults directivity effects dominate the spatial variation of the amplitudes of the ground motion. In the domain with the strike-slip fault, the peak horizontal displacements and velocities increase along the strike of the fault away from the epicenter until the end of the fault where they decay rapidly with distance. In the domain with the buried thrust fault, the largest motions occur updip from the top of the fault, and when the top of the fault is raised to the ground surface, the motions increase and become most severe on the hanging wall along the surface rupture.

Friction models with rate weakening (e.g., the slip- and rate-weakening friction model) generate pulselike ruptures with more realistic characteristics than those without rate weakening (e.g., the slip-weakening friction model), which generate cracklike ruptures. The pulselike ruptures tend to create heterogeneity in the distributions of the peak slip rate and the final slip while maintaining heterogeneity in the shear tractions. The slip-weakening friction model, on the other hand, tends to create heterogeneity only in the distribution of the maximum slip rate while reducing the heterogeneity in the shear tractions. Furthermore, for surface ruptures on a thrust fault, the friction models with rateweakening behavior limit the dynamic interaction between the seismic waves reflected off the ground surface and the propagating rupture. Whereas the ground motions remain more severe on the hanging wall of the fault, the radiated energy follows the Gutenberg-Richter relationship, and the ground motions do not reach the extreme levels that they do with the slip-weakening friction model. 


\section{Acknowledgments}

This work was supported by a National Science Foundation fellowship, the Southern California Earthquake Center, and the California Institute of Technology. Access to the Hewlett-Packard X-Class computer, located at the California Institute of Technology, was provided by the Center for Advanced Computing Research. We appreciate the helpful reviews from Steven Day, Ruth Harris, and David Oglesby.

\section{References}

Aagaard, B. T. (1999). Finite-element simulations of earthquakes, Technical report 99-03, California Institute of Technology, Earthquake Engineering Research Laboratory, Pasadena, California.

Aagaard, B. T., J. F. Hall, and T. H. Heaton (2001). Characterization of near-source ground motions with earth-quake simulations, Earthquake Spectra 17, no. 2, 177-207.

Andrews, D. (1976a). Rupture propagation with finite stress in antiplane strain, J. Geophys. Res. 81, no. 20, 3575-3582.

Andrews, D. (1976b). Rupture velocity of plane strain shear cracks, J. Geophys. Res. 81, no. 32, 5679-5687.

Anooshehpoor, A., and J. Brune (1999). Wrinkle-like Weertman pulse at the interface between two blocks of foam rubber with different velocities, Geophys. Res. Lett. 26, no. 13, 2025-2028.

Archuleta, R. J. (1984). A faulting model for the 1979 Imperial-Valley earthquake, J. Geophys. Res.-Solid Earth 89, no. B6, 4559-4585.

Archuleta, R. J., and S. M. Day (1980). Dynamic rupture in a layered medium: the 1966 Parkfield earthquake, Bull. Seism. Soc. Am. 70, no. 3, 671-689.

Ben-Zion, Y., and D. Andrews (1998). Properties and implications of dynamic rupture along a material interface, Bull. Seism. Soc. Am. 88, no. $4,1085-1094$.

Beroza, G., and T. Mikumo (1996). Short slip duration in dynamic rupture in the presence of heterogeneous fault properties, J. Geophys. Res.Solid Earth 101, no. B10, 22,449-22,460.

Beroza, G. C., and P. Spudich (1988). Linearized inversion for fault rupture behavior-application to the 1984 Morgan-Hill, California, earthquake, J. Geophys. Res.-Solid Earth 93, no. B6, 6275-6296.

Bouchon, M. (1997). The state of stress on some faults of the San Andreas system as inferred from near-field strong motion data, J. Geophys. Res.-Solid Earth 102, no. B6, 11,731-11,744.

Brodsky, E. E., and H. Kanamori (2001). Elastohydrodynamic lubrication of faults, J. Geophys. Res. 106, no. 86, 16,357-16,374.

Brune, J. N. (1996). Particle motion in a physical model of shallow angle thrust faulting, Proc. Indian Acad. Sci.: Earth Planet. Sci. 105, no. 2, L197-L206.

Brune, J., S. Brown, and P. Johnson (1993). Rupture mechanism and interface separation in foam rubber models of earthquakes-a possible solution to the heat-flow paradox and the paradox of large overthrusts, Tectonophysics 218, no. 1-3, 59-67.

Brune, J. N., T. L. Henyey, and R. F. Roy (1969). Heat flow, stress, and the rate of slip along the San Andreas fault, California, J. Geophys. Res. 74, no. 15, 3821-3827.

Burridge, R., G. Conn, and L. Freund (1979). The stability of a rapid mode II shear crack with finite cohesive traction, J. Geophys. Res.-Solid Earth 85, no. B5, 2210-2222.

Chester, F., and J. Chester (1998). Ultracataclasite structure and friction processes of the Punchbowl fault, San Andreas system, California, Tectonophysics 295, no. 1-2, 199-221.

Dahlen, F. (1977). The balance of energy in earthquake faulting, Geophys. J. R. Astr. Soc. 48, 239-261.

Day, S. M. (1982a). Three-dimensional finite-difference simulation of fault dynamics: rectangular faults with fixed rupture velocity, Bull. Seism. Soc. Am. 72, no. 3, 705-727.

Day, S. M. (1982b). Three-dimensional simulation of spontaneous rupture: the effect of nonuniform prestress, Bull. Seism. Soc. Am. 72, no. 6, 1881-1902.

Day, S. M., G. Yu, and D. J. Wald (1998). Dynamic stress change during earthquake rupture, Bull. Seism. Soc. Am. 88, no. 2, 512-522.

Dieterich, J. (1992). Earthquake nucleation on faults with rate-dependent and state-dependent strength, Tectonophysics 211, no. 1-4, 115-134.

Freund, L. (1979). The mechanics of dynamic shear crack propagation, $J$. Geophys. Res.-Solid Earth 84, no. B5, 2199-2209.

Freund, L. (1990). Dynamic fracture mechanics, in Cambridge Monographs on Mechanics and Applied Mathematics, Cambridge University Press, New York.

Fukuyama, E., and R. Madariaga (1998). Rupture dynamics of a planar fault in a 3-D elastic medium: rate- and slip-weakening friction, Bull. Seism. Soc. Am. 88, no. 1, 1-17.

Guatteri, M., and P. Spudich (1998). Coseismic temporal changes of slip direction: the effect of absolute stress on dynamic rupture, Bull. Seism. Soc. Am. 88, no. 3, 777-789.

Guatteri, M., and P. Spudich (2000). What can strong-motion data tell us about slip-weakening fault-friction laws? Bull. Seism. Soc. Am. 90, no. $1,98-116$.

Hall, J. F., T. H. Heaton, M. W. Halling, and D. J. Wald (1995). Nearsource ground motion and its effects on flexible buildings, Earthquake Spectra 11, no. 4, 569-605.

Harris, R., and S. Day (1997). Effects of a low-velocity zone on a dynamic rupture, Bull. Seism. Soc. Am. 87, no. 5, 1267-1280.

Harris, R., and S. Day (1999). Dynamic 3-D simulations of earthquakes on en echelon faults, Geophys. Res. Lett. 26, no. 14, 2089-2092.

Haskell, N. (1969). Elastic displacements in the near-field of a propagating fault, Bull. Seism. Soc. Am. 59, no. 2, 865-908.

Heaton, T. H. (1990). Evidence for and implications of self-healing pulses of slip in earthquake rupture, Phys. Earth Planet. Interiors 64, no. 1, $1-20$.

Heaton, T. H., F. Tajima, and A. Mori (1986). Estimating ground motions using recorded accelerograms, Surv. Geophys. 8, no. 1, 25-83.

Hernandez, B., F. Cotton, and M. Campillo (1999). Contribution of radar interferometry to a two-step inversion of the kinematic process of the 1992 Landers earthquake, J. Geophys. Res.-Solid Earth 104, no. B6, 13,083-13,099.

Ida, Y. (1972). Cohesive force across the tip of a longitudinal-shear crack and Griffith's specific surface energy, J. Geophys. Res. 77, no. 20, 3796-3805.

Kanamori, H., and T. H. Heaton (2000). Microscopic and macroscopic physics of earthquakes, in Geocomplexity and the Physics of Earthquakes, J. B. Rundle (Editor), Vol. 120, American Geophysical Union, Washington, D.C., 147-163.

Kanamori, H., D. L. Anderson, and T. H. Heaton (1998). Frictional melting during the rupture of the 1994 Bolivian earthquake, Science 279, 839842.

Karypis, G., R. Aggarwal, V. Kumar, and S. Shekhar (1999). Multilevel hypergraph partitioning: applications in VLSI domain, IEEE Trans. Very Large Scale Integr. (VLSI) Syst. 7, no. 1, 69-79.

Lachenbruch, A. (1980). Friction heating, fluid pressure, and the resistance to fault motion, J. Geophys. Res.-Solid Earth 85, no. B11, 6097-6112.

Lawn, B. R. (1993). Fracture of Brittle Solids, Second Ed., Cambridge University Press, New York.

Madariaga, R. (1976). Dynamics of an expanding circular fault, Bull. Seism. Soc. Am. 66, no. 3, 639-666.

Madariaga, R., and A. Cochard (1996). Dynamic friction and the origin of the complexity of earthquake sources, Proc. Natl. Acad. Sci. USA 93, no. 9, 3819-3824.

Madariaga, R., K. Olsen, and R. Archuleta (1998). Modeling dynamic rupture in a 3-D earthquake fault model, Bull. Seism. Soc. Am. 88, no. 5, 1182-1197.

Magistrale, H., and S. Day (1999). 3-D simulations of multi-segment thrust fault rupture, Geophys. Res. Lett. 26, no. 14, 2093-2096.

Marone, C. (1998). Laboratory-derived friction laws and their application to seismic faulting, Annu. Rev. Earth Planet. Sci. 26, 643-696. 
McKenzie, D., and J. Brune (1972). Melting on fault planes during large earthquakes, Geophys. J. R. Astr. Soc. 29, 65-78.

Melosh, H. (1996). Dynamical weakening of faults by acoustic fluidization, Nature 379, no. 6566, 601-606.

Mikumo, T. (1992). Dynamic fault rupture and stress recovery processes in continental crust under depth-dependent shear strength and frictional parameters, Tectonophysics 211, no. 1-4, 201-222.

Mikumo, T., and T. Miyatake (1993). Dynamic rupture processes on a dipping fault, and estimates of stress drop and strength excess from the results of wave-form inversion, Geophys. J. Int. 112, no. 3, 481496.

Nielsen, S., and K. Olsen (2000). Constraints on stress and friction from dynamic rupture models of the 1994 Northridge, California, earthquake, Pure Appl. Geophys. 157, no. 11-12, 2029-2046.

Oglesby, D. D., R. J. Archuleta, and S. B. Nielsen (1998). Earthquakes on dipping faults: the effect of broken symmetry, Science 280, no. 5366, 1055-1059.

Oglesby, D. D., R. J. Archuleta, and S. B. Nielsen (2000a). Dynamics of dip-slip faulting: explorations in two dimensions, J. Geophys. Res.Solid Earth 105, no. B6, 13,643-13,653.

Oglesby, D. D., R. J. Archuleta, and S. B. Nielsen (2000b). The threedimensional dynamics of dipping faults, Bull. Seism. Soc. Am. 90, no. 3, 616-628.

Olsen, K., and R. Archuleta (1996). Three-dimensional simulation of earthquakes on the Los Angeles fault system, Bull. Seism. Soc. Am. 86, no. 3, 575-596.

Olsen, K., R. Madariaga, and R. Archuleta (1997). Three-dimensional dynamic simulation of the 1992 Landers earthquake, Science 278, 834839.

Parsons, I., J. Hall, and G. Lyzenga (1988). Relationships between the average offset and the stress drop for two-dimensional and threedimensional faults, Bull. Seism. Soc. Am. 78, no. 2, 931-945.

Persson, B. N. (1997). Sliding friction: physical principles and applications, in NanoScience and Technology, Springer, New York.

Rice, J. (1983). Constitutive relations for fault slip and earthquake instabilities, Pure Appl. Geophys. 121, no. 3, 443-475.

Rice, J. R., and Y. Ben-Zion (1996). Slip complexity in earthquake fault models, Proc. Natl. Acad. Sci. USA 93, no. 9, 3811-3818.

Richards, P. G. (1976). Dynamic motions near an earthquake fault: a threedimensional solution, Bull. Seism. Soc. Am. 66, no. 1, 1-32.

Rosakis, A., O. Samudrala, and D. Coker (1999). Cracks faster than the shear wave speed, Science 284, no. 5418, 1337-1340.

Savage, J., and J. Walsh (1978). Gravitational energy and faulting, Bull. Seism. Soc. Am. 68, no. 6, 1613-1622.

Scholz, C. H. (1998). Earthquakes and friction laws, Nature 391, no. 6662, $37-42$.

Shi, B., A. Anooshehpoor, J. N. Brune, and Y. Zeng (1998). Dynamics of thrust faulting: 2-D lattice model, Bull. Seism. Soc. Am. 88, no. 6, 1484-1494.

Sleep, N. H. (1997). Application of a unified rate and state friction theory to the mechanics of fault zones with strain localization, J. Geophys. Res.-Solid Earth 102, no. B2, 2875-2895.

Somerville, P. G., K. Irikura, R. Graves, S. Sawada, D. Wald, N. Abrahamson, Y. Iwasaki, T. Kagawa, N. Smith, and A. Kowada (1999). Characterizing crustal earthquake slip models for the prediction of strong ground motion, Seism. Res. Lett. 70, no. 1, 199-222.

Steketee, J. (1958). Some geophysical applications of the elasticity theory of dislocations, Can. J. Phys. 36, 1168-1198.

Stuart, W. D., and G. M. Mavko (1979). Earthquake instability on a strikeslip fault, J. Geophys. Res.-Solid Earth 84, no. B5, 2153-2160.
Tullis, T. E. (1996). Rock friction and its implications for earthquake prediction examined via models of Parkfield earthquakes, Proc. Natl. Acad. Sci. USA 93, no. 9, 3803-3810.

Tworzydlo, W. W., and O. N. Hamzeh (1997). On the importance of normal vibrations in modeling of stick slip in rock sliding, J. Geophys. Res.Solid Earth 10, no. B7, 15,091-15,103.

\section{Appendix}

\section{Boundary Conditions and Energy Balance}

Steketee (1958) noted that for an elastic half-space under constant loading, the strain energy of the half-space increases when slip occurs on a fault as a result of the loading. However, the Earth is a finite body under the force of gravity, and the tectonic forces driving the dislocations change as a result of earthquakes. In modeling only a small fraction of the Earth, the tectonic forces do not receive the information about the dislocation until the rupture has completed. This means that the tectonic forces on the boundary remain constant during the rupture.

Holding the tectonic forces on the boundaries constant affects the energy balance because the boundaries can do work during the earthquake as the boundary deforms. However, we formulate the energy balance considering only the radiated energy, the change in thermal energy, and the change in potential energy. Because the sliding is completed before the tectonic forces can change, any work done by the boundary affects neither the radiated energy nor the change in thermal energy (which we associate directly with the rupture and the stresses on the fault during the sliding). Consequently, it does not affect the total change in potential energy either; it only affects the partitioning of the change in potential energy among the different forms of potential energy, such as strain energy and gravitational potential energy. Thus, our choice of keeping the tectonic forces constant on the boundary does not affect the computation of the radiated energy, the change in thermal energy, or the change in potential energy.

Department of Geologic and Planetary Sciences

Mail Stop 252-21

California Institute of Technology

Pasadena, California 91125

(B.T.A., T.H.H.)

Department of Civil Engineering

Mail Stop 104-44

California Institute of Technology

Pasadena, California 91125

(J.F.H.) 